\title{
GEGENBAUER WAVELETS OPERATIONAL MATRIX METHOD FOR FRACTIONAL DIFFERENTIAL EQUATIONS
}

\author{
Mujeeb ur Rehman and Umer Saeed
}

\begin{abstract}
In this article we introduce a numerical method, named Gegenbauer wavelets method, which is derived from conventional Gegenbauer polynomials, for solving fractional initial and boundary value problems. The operational matrices are derived and utilized to reduce the linear fractional differential equation to a system of algebraic equations. We perform the convergence analysis for the Gegenbauer wavelets method.

We also combine Gegenbauer wavelets operational matrix method with quasilinearization technique for solving fractional nonlinear differential equation. Quasilinearization technique is used to discretize the nonlinear fractional ordinary differential equation and then the Gegenbauer wavelet method is applied to discretized fractional ordinary differential equations. In each iteration of quasilinearization technique, solution is updated by the Gegenbauer wavelet method. Numerical examples are provided to illustrate the efficiency and accuracy of the methods.
\end{abstract}

\section{Introduction}

Fractional differential equation is the generalization of the ordinary differential equation and is used to model the problems in many areas of science and engineering such as fluid mechanics [19], dynamic of viscoelastic materials [20], biosciences [23], electromagnetism [15] and continuum and statistical mechanics [24]. For most of the fractional differential equations, exact solutions are not known. Transform methods can be used to solve fractional differential equations exactly, but their applicability is limited to certain classes of linear fractional differential equations. Therefore different numerical method for providing approximate solutions to classical differential equations are extended to solve fractional differential equations. Some of these techniques include, fractional difference method [25], differential transform method [2], Adomian decomposition method [14] and homotopy analysis method [16].

The wavelet analysis is the decomposition of a function onto shifted and scaled versions of the basic wavelet. The wavelet basis are orthogonal basis for

Received December 23, 2014; Revised January 19, 2015.

2010 Mathematics Subject Classification. 65L60, 65M70, 65N35.

Key words and phrases. Gegenbauer polynomials, Gegenbauer wavelets, operational matrices, fractional differential equations, convergence analysis, quasilinearization. 
$L_{2}(\mathbb{R})$ and are generated by the translation and dilatation of the basic wavelet. They are compactly supported. The Haar wavelet $[21,31]$ is the simplest of the orthonormal wavelets with compact support and was constructed by Haar in 1909. Haar wavelet operational matrix of integration was first derived by Chen et al. [7] to solve the differential equations. There are several other wavelets which can be used to solve the differential equations. Some of these include, Daubechies [8, 32], B-spline [9], Legendre [29, 35], Hermite [1, 33] and Chebyshev [3, 17]. Legendre, Hermite and Chebyshev wavelets uses Legendre, Hermite and Chebyshev polynomials as their basis functions, respectively.

Linearization is carried out by considering the first two terms in the Taylor's series expansion of the original nonlinear differential equation. This technique is a generalized Newton-Raphson method for functional equations. It is also known as the quasilinearization method. The quasilinearization technique not only linearizes the nonlinear equation but also provides a sequence of functions which in general converges rather rapidly to the solution of the original nonlinear equation. The main advantage of this technique is that the procedure converges quadratically to the solution of the original equation, if there is convergence at all. The method of quasilinearization developed by Bellman $[4,5]$ and was first used to obtain a representation for the solution of the initial value problem for the Riccati equation. Bellman and Kalaba [6] generalized these results and obtained a solution formula for a wide class of nonlinear first order differential equations. Mohapatra et al. [26] established the existence, uniqueness, and convergence results for general second order nonlinear boundary value problems by using quasilinearization and monotone iterative methods. Devi et al. [11] developed the method of quasilinearization for fractional differential equations. Existence and uniqueness result for an initial value problem of fractional differential equations using generalized quasilinearization technique is obtained in [10].

Gegenbauer polynomials [12] or ultraspherical polynomials are orthogonal polynomials on the interval $[-1,1]$ with respect to the weight function. They generalize the Legendre and Chebyshev polynomials. In the present work, we constructed the Gegenbauer wavelets by using the shifted Gegenbauer polynomials as their basis functions. The interval $[0,1)$ is chosen to be the compact support of Gegenbauer wavelets. The purpose of introducing the Gegenbauer wavelets is to develop a numerical method for solving fractional differential equations, which we named Gegenbauer wavelets method. Operational matrices are derived and utilized for solving fractional initial and boundary value problems. Boundary value problems are considerably more difficult to deal with than the initial value problems. Gegenbauer wavelets method for boundary value problems is more complicated than for initial value problems. We need additional operational matrices for tackling the boundary conditions while solving boundary value problems. We first discritize the nonlinear differential equation by quasilinearization technique and then implement the Gegenbauer 
wavelet method on discretized differential equation. At each iteration of quasilinearization technique solution is updated by Gegenbauer wavelet method. The method is implement on number of problems and numerical results are compared with solutions obtained by some other numerical techniques [30, 37] and exact solutions as well.

\section{Preliminaries}

We review basic definitions of fractional differentiation and fractional integration [27]:

1) Riemann-Liouville fractional integral operator of order $\alpha$ :

The Riemann-Liouville fractional order integral of order $\alpha \in \mathbb{R}^{+}$is defined as

$$
I_{a}^{\alpha} y(x)=\frac{1}{\Gamma(\alpha)} \int_{a}^{x}(x-t)^{\alpha-1} y(t) d t
$$

for $a<x \leq b$, and $I_{a}^{\alpha}$ becomes zero for $x=a$.

2) Riemann-Liouville and Caputo fractional derivative operator of order $\alpha$ :

The Riemann-Liouville fractional order derivative of order $\alpha \in \mathbb{R}^{+}$is defined as

$$
{ }_{R} D_{a}^{\alpha} y(x)=\frac{1}{\Gamma(n-\alpha)}\left(\frac{d}{d x}\right)^{n} \int_{a}^{x}(x-t)^{n-\alpha-1} y(t) d t
$$

for $a<x \leq b$, where $n-1<\alpha<n, n \in \mathbb{N}$ and $n=\lceil\alpha\rceil$.

The Caputo fractional order derivative of order $\alpha \in \mathbb{R}^{+}$is defined as

$$
{ }_{C} D_{a}^{\alpha} y(x)=\frac{1}{\Gamma(n-\alpha)} \int_{a}^{x}(x-t)^{n-\alpha-1}\left(\frac{d}{d t}\right)^{n} y(t) d t
$$

for $a<x \leq b$, where $n-1<\alpha<n, n \in \mathbb{N}$ and $n=\lceil\alpha\rceil . \quad{ }_{R} D_{a}^{\alpha}$ and ${ }_{C} D_{a}^{\alpha}$ becomes zero for $x=a$.

\section{Gegenbauer polynomials and Gegenbauer wavelets}

The Gegenbauer polynomials [12], or ultra spherical harmonics polynomials, $C_{m}^{\lambda}(x)$, of order $m$ are defined, for $\lambda>-\frac{1}{2}, m \in \mathbb{Z}^{+}$, on the interval $[-1,1]$ and given by the following recurrence formulae,

$C_{0}^{\lambda}(x)=1, C_{1}^{\lambda}(x)=2 \lambda x$,

$C_{m+1}^{\lambda}(x)=\frac{1}{m+1}\left(2(m+\lambda) x C_{m}^{\lambda}(x)-(m+2 \lambda-1) C_{m-1}^{\lambda}(x)\right), m=1,2,3, \ldots$

The Gegenbauer polynomials are orthogonal on $[-1,1]$ with respect to the weight function $w(x)=\left(1-x^{2}\right)^{\lambda-\frac{1}{2}}$ as

$$
\int_{-1}^{1}\left(1-x^{2}\right)^{\lambda-\frac{1}{2}} C_{m}^{\lambda}(x) C_{n}^{\lambda}(x) d x=L_{m}^{\lambda} \delta_{m n}, \quad \lambda>-\frac{1}{2},
$$


where $L_{m}^{\lambda}(x)=\frac{\pi 2^{1-2 \lambda} \Gamma(m+2 \lambda)}{m !(m+\lambda)(\Gamma(\lambda))^{2}}$ is the normalizing factor and $\delta$ is the Kronecker delta function.

Gegenbauer polynomials generalize Legendre polynomials and Chebyshev polynomials. For $\lambda=0$ and $\lambda=1$, we get Chebyshev polynomials of first and second kind respectively and at $\lambda=\frac{1}{2}$ we get Legendre polynomial.

Scaling and translation of the basic wavelet (mother wavelet) $\psi(x)$ define the basis

$$
\psi_{p, q}(x)=\frac{1}{\sqrt{|p|}} \psi\left(\frac{x-q}{p}\right), p, q \in \mathbb{R}, p \neq 0,
$$

where $p$ is scaling parameter and $q$ is the translation parameter. By restricting $p, q$ to discrete values as: $p=p_{0}^{-k}, q=n q_{0} p_{0}^{-k}$, where $p_{0}>1, q_{0}>0$ and $k, n \in \mathbb{N}$, we get the following family of discrete wavelets as

$$
\psi_{k, n}(x)=\left(p_{0}\right)^{\frac{k}{2}} \psi\left(p_{0}^{k} x-n q_{0}\right)
$$

The set of wavelets forms an orthogonal basis of $L_{2}(\mathbb{R})$. In particular, when $p_{0}=2$ and $q_{0}=1$, then $\psi_{k, n}$ forms an orthonormal basis. That is

$$
\left\langle\psi_{k, n}(x), \psi_{l, m}(x)\right\rangle=\delta_{k l} \delta_{n m} .
$$

The discrete wavelets transform is defined as

$$
\psi_{k, n}(x)=(2)^{\frac{k}{2}} \psi\left(2^{k} x-n\right) .
$$

The Gegenbauer wavelets are defined on interval $[0,1)$ by

$$
\psi_{n, m}^{\lambda}(x)= \begin{cases}\frac{1}{\sqrt{L_{m}^{\lambda}}} 2^{\frac{k}{2}} C_{m}^{\lambda}\left(2^{k} x-\hat{n}\right), & \frac{\hat{n}-1}{2^{k}} \leq t<\frac{\hat{n}+1}{2^{k}} \\ 0, & \text { elsewhere }\end{cases}
$$

where $k=1,2,3, \ldots$, is the level of resolution, $n=1,2,3, \ldots, 2^{k-1}, \hat{n}=2 n-$ 1 , is the translation parameter, $m=0,1,2, \ldots, M-1$ is the order of the Gegenbauer polynomials, $M>0$. Corresponding to each $\lambda>-\frac{1}{2}$, we have a different family of wavelets, i.e., when $\lambda=\frac{1}{2}$, we get Legendre wavelets [28], $\psi_{n, m}^{\frac{1}{2}}(x)$. Similarly for $\lambda=0$ and $\lambda=1$, we obtain the Chebyshev wavelet of first [22] and second kind [36], respectively. In the present work, we utilize the Gegenbauer wavelets at different values of $\lambda>-\frac{1}{2}$.

It is to be noted that the weight function for the Gegenbauer polynomials have to be dilated and translated for the Gegenbauer wavelets, in order to obtain the orthogonality of wavelets, as

$$
w_{n, k}(x)=w\left(2^{k} x-2 n+1\right)=\left(1-\left(2^{k} x-2 n+1\right)^{2}\right)^{\lambda-\frac{1}{2}} .
$$


At fixed level of resolution $k=p$ (say), we get

$$
w_{n, p}(x)=\left\{\begin{array}{cc}
w_{1, p}(x), & 0 \leq x<\frac{1}{2^{p-1}} \\
w_{2, p}(x), & \frac{1}{2^{p-1}} \leq x<\frac{2}{2^{p-1}} \\
w_{3, p}(x), & \frac{2}{2^{p-1}} \leq x<\frac{3}{2^{p-1}} \\
\vdots & \\
w_{2^{p-1}, p}(x), & \frac{2^{p-1}-1}{2^{p-1}} \leq x<1 .
\end{array}\right.
$$

\section{Function approximations and the Gegenbauer wavelets matrix}

We can expand any function $f(x) \in L^{2}[0,1)$ into truncated Gegenbauer wavelet series as,

$$
f(x) \approx \sum_{n=1}^{2^{k-1}} \sum_{m=0}^{M-1} a_{n m}^{\lambda} \psi_{n, m}^{\lambda}(x)=\mathbf{a}^{\lambda^{T}} \boldsymbol{\Psi}^{\lambda}(x),
$$

where $\mathbf{a}^{\lambda}$ and $\Psi^{\lambda}$ are $\hat{m} \times 1,\left(\hat{m}=2^{k-1} M\right)$, matrices, given by

$$
\begin{gathered}
\mathbf{a}^{\lambda}=\left[a_{10}^{\lambda}, a_{11}^{\lambda}, \ldots, a_{1 M-1}^{\lambda}, a_{20}^{\lambda}, a_{21}^{\lambda}, \ldots, a_{2 M-1}^{\lambda}, \ldots, a_{2^{k-1} 0}^{\lambda}, a_{2^{k-1} 1}^{\lambda}, \ldots,\right. \\
\left.\quad a_{2^{k-1} M-1}^{\lambda}\right]^{T}, \\
\boldsymbol{\Psi}^{\lambda}(x)=\left[\psi_{1,0}^{\lambda}(x), \psi_{1,1}^{\lambda}(x), \ldots, \psi_{1 M-1}^{\lambda}(x), \psi_{2,0}^{\lambda}(x), \psi_{2,1}^{\lambda}(x), \ldots, \psi_{2, M-1}^{\lambda}(x), \ldots,\right. \\
\left.\psi_{2^{k-1}, 0}^{\lambda}(x), \psi_{2^{k-1}, 1}^{\lambda}(x), \ldots, \psi_{2^{k-1}, M-1}^{\lambda}(x)\right]^{T} .
\end{gathered}
$$

The collocation points for the Gegenbauer wavelets are taken as $x_{i}=\frac{2 i-1}{2^{k} M}$, where $i=1,2, \ldots, 2^{k-1} M$. The Gegenbauer wavelet matrix $\Psi^{\lambda} 2^{k-1} M, 2^{k-1} M$ is given as

$$
\boldsymbol{\Psi}_{2^{k-1} M \times 2^{k-1} M}^{\lambda}=\left[\boldsymbol{\Psi}^{\lambda}\left(\frac{1}{2^{k} M}\right), \boldsymbol{\Psi}^{\lambda}\left(\frac{3}{2^{k} M}\right), \ldots, \boldsymbol{\Psi}^{\lambda}\left(\frac{2^{k} M-1}{2^{k} M}\right)\right]
$$

or

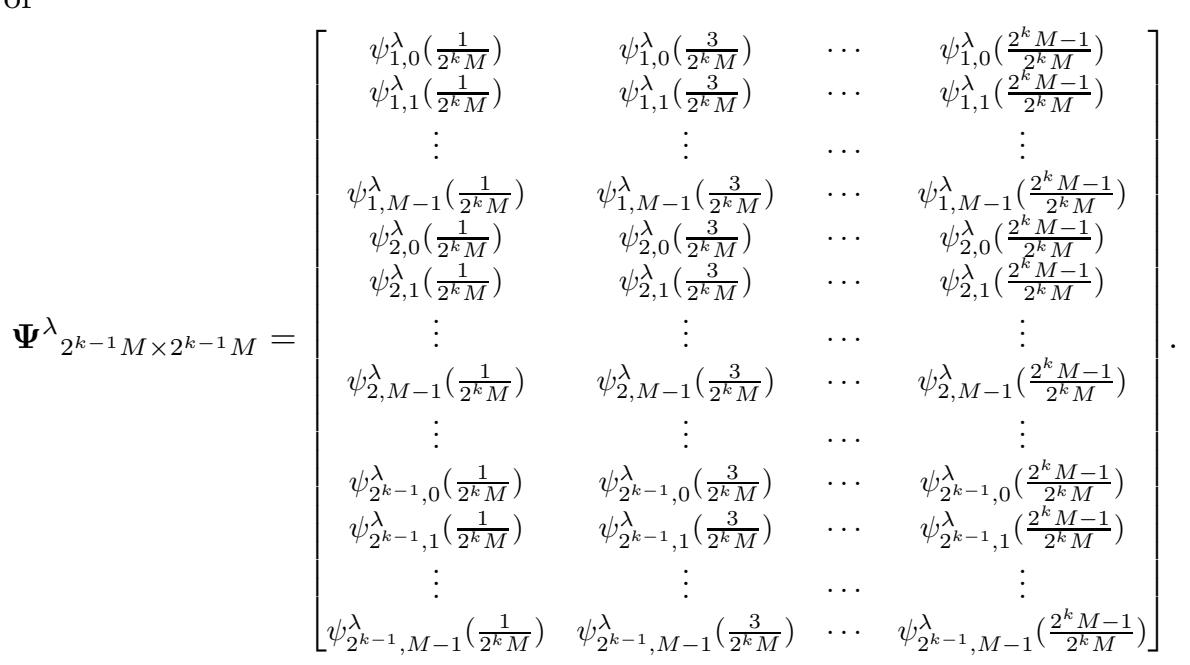


In particular, we fix $k=2, M=3$, we have $n=1,2$ and $m=0,1,2$, the Gegenbauer wavelet matrix is given as

$$
\boldsymbol{\Psi}_{6 \times 6}^{\lambda}=\left[\begin{array}{llllll}
\psi_{1,0}^{\lambda}\left(\frac{1}{12}\right) & \psi_{1,0}^{\lambda}\left(\frac{3}{12}\right) & \psi_{1,0}^{\lambda}\left(\frac{5}{12}\right) & \psi_{1,0}^{\lambda}\left(\frac{7}{12}\right) & \psi_{1,0}^{\lambda}\left(\frac{9}{12}\right) & \psi_{1,0}^{\lambda}\left(\frac{11}{12}\right) \\
\psi_{2,0}^{\lambda}\left(\frac{1}{12}\right) & \psi_{2,0}^{\lambda}\left(\frac{3}{12}\right) & \psi_{2,0}^{\lambda}\left(\frac{5}{12}\right) & \psi_{2,0}^{\lambda}\left(\frac{7}{12}\right) & \psi_{2,0}^{\lambda}\left(\frac{9}{12}\right) & \psi_{2,0}^{\lambda}\left(\frac{11}{12}\right) \\
\psi_{1,1}^{\lambda}\left(\frac{1}{12}\right) & \psi_{1,1}^{\lambda}\left(\frac{3}{12}\right) & \psi_{1,1}^{\lambda}\left(\frac{5}{12}\right) & \psi_{1,1}^{\lambda}\left(\frac{7}{12}\right) & \psi_{1,1}^{\lambda}\left(\frac{9}{12}\right) & \psi_{1,1}^{\lambda}\left(\frac{11}{12}\right) \\
\psi_{2,1}^{\lambda}\left(\frac{1}{12}\right) & \psi_{2,1}^{\lambda}\left(\frac{3}{12}\right) & \psi_{2,1}^{\lambda}\left(\frac{5}{12}\right) & \psi_{2,1}^{\lambda}\left(\frac{7}{12}\right) & \psi_{2,1}^{\lambda}\left(\frac{9}{12}\right) & \psi_{2,1}^{\lambda}\left(\frac{11}{12}\right) \\
\psi_{1,2}^{\lambda}\left(\frac{1}{12}\right) & \psi_{1,2}^{\lambda}\left(\frac{3}{12}\right) & \psi_{1,2}^{\lambda}\left(\frac{5}{12}\right) & \psi_{1,2}^{\lambda}\left(\frac{7}{12}\right) & \psi_{1,2}^{\lambda}\left(\frac{9}{12}\right) & \psi_{1,2}^{\lambda}\left(\frac{11}{12}\right) \\
\psi_{2,2}^{\lambda}\left(\frac{1}{12}\right) & \psi_{2,2}^{\lambda}\left(\frac{3}{12}\right) & \psi_{2,2}^{\lambda}\left(\frac{5}{12}\right) & \psi_{2,2}^{\lambda}\left(\frac{7}{12}\right) & \psi_{2,2}^{\lambda}\left(\frac{9}{12}\right) & \psi_{2,2}^{\lambda}\left(\frac{11}{12}\right)
\end{array}\right]
$$

Compact support of the Gegenbauer wavelets is $\left[\frac{2 n-2}{2^{k}}, \frac{2 n}{2^{k}}\right)$, when $n=1$, support of $\psi_{1, m}^{\lambda}(x)$ is $\left[0, \frac{1}{2}\right)$, so $\psi_{1,0}^{\lambda}(x), \psi_{1,1}^{\lambda}(x), \psi_{1,2}^{\lambda}(x)$ are zero at $x=\frac{7}{12}, \frac{9}{12}$, $\frac{11}{12}$. Similarly, for $n=2$, support of $\psi_{2, m}^{\lambda}(x)$ is $\left[\frac{1}{2}, 1\right)$, this implies that $\psi_{2,0}^{\lambda}(x)$, $\psi_{2,1}^{\lambda}(x), \psi_{2,2}^{\lambda}(x)$ are zero at $x=\frac{1}{12}, \frac{3}{12}, \frac{5}{12}$, we have

$$
\boldsymbol{\Psi}_{6 \times 6}^{\lambda}=\left[\begin{array}{cccccc}
\psi_{1,0}^{\lambda}\left(\frac{1}{12}\right) & \psi_{1,0}^{\lambda}\left(\frac{3}{12}\right) & \psi_{1,0}^{\lambda}\left(\frac{5}{12}\right) & 0 & 0 & 0 \\
0 & 0 & 0 & \psi_{2,0}^{\lambda}\left(\frac{7}{12}\right) & \psi_{2,0}^{\lambda}\left(\frac{9}{12}\right) & \psi_{2,0}^{\lambda}\left(\frac{11}{12}\right) \\
\psi_{1,1}^{\lambda}\left(\frac{1}{12}\right) & \psi_{1,1}^{\lambda}\left(\frac{3}{12}\right) & \psi_{1,1}^{\lambda}\left(\frac{5}{12}\right) & 0 & 0 & 0 \\
0 & 0 & 0 & \psi_{2,1}^{\lambda}\left(\frac{7}{12}\right) & \psi_{2,1}^{\lambda}\left(\frac{9}{12}\right) & \psi_{2,1}^{\lambda}\left(\frac{11}{12}\right) \\
\psi_{1,2}^{\lambda}\left(\frac{1}{12}\right) & \psi_{1,2}^{\lambda}\left(\frac{3}{12}\right) & \psi_{1,2}^{\lambda}\left(\frac{5}{12}\right) & 0 & 0 & 0 \\
0 & 0 & 0 & \psi_{2,2}^{\lambda}\left(\frac{7}{12}\right) & \psi_{2,2}^{\lambda}\left(\frac{9}{12}\right) & \psi_{2,2}^{\lambda}\left(\frac{11}{12}\right)
\end{array}\right]
$$

For fix value of $\lambda$, say $\lambda=5$, we have

$$
\boldsymbol{\Psi}_{6 \times 6}^{\mathbf{5}}=\left[\begin{array}{cccccc}
\frac{2}{\sqrt{L_{0}^{5}}} C_{0}^{5}\left(\frac{-2}{3}\right) & \frac{2}{\sqrt{L_{0}^{5}}} C_{0}^{5}(0) & \frac{2}{\sqrt{L_{0}^{5}}} C_{0}^{5}\left(\frac{2}{3}\right) & 0 & 0 & 0 \\
0 & 0 & 0 & \frac{2}{\sqrt{L_{0}^{5}}} C_{0}^{5}\left(\frac{-2}{3}\right) & \frac{2}{\sqrt{L_{0}^{5}}} C_{0}^{5}(0) & \frac{2}{\sqrt{L_{0}^{5}}} C_{0}^{5}\left(\frac{2}{3}\right) \\
\frac{2}{\sqrt{L_{1}^{5}}} C_{1}^{5}\left(\frac{-2}{3}\right) & \frac{2}{\sqrt{L_{1}^{5}}} C_{1}^{5}(0) & \frac{2}{\sqrt{L_{1}^{5}}} C_{1}^{5}\left(\frac{2}{3}\right) & 0 & 0 & 0 \\
0 & 0 & 0 & \frac{2}{\sqrt{L_{1}^{5}}} C_{1}^{5}\left(\frac{-2}{3}\right) & \frac{2}{\sqrt{L_{1}^{5}}} C_{1}^{5}(0) & \frac{2}{\sqrt{L_{1}^{5}}} C_{1}^{5}\left(\frac{2}{3}\right) \\
\frac{2}{\sqrt{L_{2}^{5}}} C_{2}^{5}\left(\frac{-2}{3}\right) & \frac{2}{\sqrt{L_{2}^{5}}} C_{2}^{5}(0) & \frac{2}{\sqrt{L_{2}^{5}}} C_{2}^{5}\left(\frac{2}{3}\right) & 0 & 0 & 0 \\
0 & 0 & 0 & \frac{2}{\sqrt{L_{2}^{5}}} C_{2}^{5}\left(\frac{-2}{3}\right) & \frac{2}{\sqrt{L_{2}^{5}}} C_{2}^{5}(0) & \frac{2}{\sqrt{L_{2}^{5}}} C_{2}^{5}\left(\frac{2}{3}\right)
\end{array}\right]
$$

or

$$
\Psi_{6 \times 6}^{5}=\left(\begin{array}{cccccc}
2.2746 & 2.2746 & 2.2746 & 0 & 0 & 0 \\
0 & 0 & 0 & 2.2746 & 2.2746 & 2.2746 \\
-5.2530 & 0 & 5.2530 & 0 & 0 & 0 \\
0 & 0 & 0 & -5.2530 & 0 & 5.2530 \\
7.8628 & -1.8145 & 7.8628 & 0 & 0 & 0 \\
0 & 0 & 0 & 7.8628 & -1.8145 & 7.8628
\end{array}\right)
$$

Similarly, we get different Gegenbauer wavelet matrices for different value of $\lambda$. 
The Gegenbauer wavelets operational matrix of fractional order integration

For simplicity, we write (3.4) as

$$
f(x) \approx \sum_{i=1}^{\hat{m}} a_{i}^{\lambda} \psi_{i}^{\lambda}(x)=\mathbf{a}^{\lambda^{T}} \Psi^{\lambda}(x),
$$

where $a_{i}^{\lambda}=a_{m, n}^{\lambda}, \psi_{i}^{\lambda}=\psi_{m, n}^{\lambda}(x)$. The index $i$ is determined by the equation $i=M(n-1)+m+1$ and $\hat{m}=2^{k-1} M$. Also, $\mathbf{a}^{\lambda}=\left[a_{1}^{\lambda}, a_{2}^{\lambda}, \ldots, a_{\hat{m}}^{\lambda}\right]^{T}, \Psi^{\lambda}(x)=$ $\left[\psi_{1}^{\lambda}(x), \psi_{2}^{\lambda}(x), \ldots, \psi_{\hat{m}}^{\lambda}(x)\right]^{T}$.

An arbitrary function $f \in L_{2}[0,1)$, can be expanded into block-pulse functions [18] as

$$
f(x) \approx \sum_{i=0}^{\hat{m}-1} f_{i} b_{i}(x)=\mathbf{f}^{T} \mathbf{B}(x),
$$

where $f_{i}$ are the coefficients of the block-pulse function. The Gegenbauer wavelets can be expanded into $m$-set of block-pulse Functions as

$$
\boldsymbol{\Psi}^{\lambda}(x)=\boldsymbol{\Psi}_{\hat{m} \times \hat{m}}^{\lambda} \mathbf{B}(x) .
$$

The fractional integral of block-pulse function vector can be written as

$$
\left(\mathcal{I}_{0}^{\alpha} \mathbf{B}\right)(x)=\mathbf{F}_{\hat{m} \times \hat{m}}^{\alpha} \mathbf{B}(x),
$$

where $\mathbf{F}_{\hat{m} \times \hat{m}}^{\alpha}$ is given in [18] with

$$
\mathbf{P}_{\hat{m} \times \hat{m}}^{\lambda, \alpha}=\boldsymbol{\Psi}_{\hat{m} \times \hat{m}}^{\lambda} \mathbf{F}^{\alpha}\left(\boldsymbol{\Psi}_{\hat{m} \times \hat{m}}^{\lambda}\right)^{-1} .
$$

The Gegenbauer wavelets operational matrix of integration $\mathbf{P}_{\hat{m} \times \hat{m}}^{\lambda, \alpha}$ of fractional order $\alpha$ is constructed for different $\lambda>-\frac{1}{2}$ and are utilize for solving differential equations.

In particular, for $k=2, M=3, \alpha=1.25, \lambda=5$, the Gegenbauer wavelet operational matrix of fractional order integration $\mathbf{P}_{6 \times 6}^{5,1.25}$ is given by

$$
\mathbf{P}_{8 \times 8}^{5,1.25}=\left(\begin{array}{cccccc}
0.1592 & 0.4581 & 0.0551 & 0.0189 & 0.0028 & -0.0017 \\
0 & 0.1592 & 0 & 0.0551 & 0 & 0.0028 \\
-0.2462 & -0.0634 & -0.0278 & 0.0090 & 0.0247 & -0.0018 \\
0 & -0.2462 & 0 & -0.0278 & 0 & 0.0247 \\
0.3599 & 0.9284 & 0.0843 & 0.0406 & -0.0061 & -0.0041 \\
0 & 0.3599 & 0 & 0.0843 & 0 & -0.0061
\end{array}\right) .
$$

For $k=2, M=3, \alpha=1.25, \lambda=12$, we have

$$
\mathbf{P}_{6 \times 6}^{12,1.25}=\left(\begin{array}{cccccc}
0.1580 & 0.4589 & 0.0374 & 0.0128 & 0.0014 & -0.0008 \\
0 & 0.1580 & 0 & 0.0374 & 0 & 0.0014 \\
-0.3781 & -0.0922 & -0.0278 & 0.0090 & 0.0179 & -0.0013 \\
0 & -0.3781 & 0 & -0.0278 & 0 & 0.0179 \\
0.8748 & 2.2912 & 0.1490 & 0.0672 & -0.0049 & -0.0048 \\
0 & 0.8748 & 0 & 0.1490 & 0 & -0.0049
\end{array}\right) .
$$


For different values of $\lambda$, we get different families of Gegenbauer wavelets matrices and their corresponding different operational matrices of fractional order integration.

This phenomena makes calculations fast because the operational matrix $\mathbf{P}_{\hat{m} \times \hat{m}}^{\lambda, \alpha}$ contains many zero entries.

\section{Operational matrix of fractional order integration for boundary value problems}

We drive another operational matrix of fractional integration to solve the fractional boundary value problems. Let $g(x) \in L_{2}[0, b]$ be a given function, $b>0$. Then

$$
g(x) I_{0}^{\alpha} \psi_{n, m}^{\lambda}(x)=\frac{g(x)}{\Gamma(\alpha)} \int_{0}^{b}(b-s)^{\alpha-1} \psi_{n, m}^{\lambda}(s) d s .
$$

Since the Gegenbauer wavelets are supported on the intervals $\left[\frac{(2 n-2) b}{2^{k}}, \frac{2 n b}{2^{k}}\right)$, therefore

$$
\begin{aligned}
g(x) I_{b}^{\alpha} \psi_{n, m}^{\lambda}(x) & =\frac{g(x) 2^{\frac{k}{2}}}{\left.\sqrt{(} L_{m}^{\lambda}\right) \Gamma(\alpha)} \int_{\frac{(2 n-2) b}{2^{k}}}^{\frac{2 n b}{2^{k}}}(b-s)^{\alpha-1} C_{m}^{\lambda}\left(2^{k} s-2 n+1\right) d s \\
& =g(x) V_{n, m}^{\lambda, \alpha, b}
\end{aligned}
$$

where $V_{n, m}^{\lambda, \alpha, b}=\frac{2^{\frac{k}{2}}}{\left.\sqrt{(} L_{m}^{\lambda}\right) \Gamma(\alpha)} \int_{\frac{(2 n-2) b}{2^{k}}}^{\frac{2 n b}{2^{k}}}(b-s)^{\alpha-1} C_{m}^{\lambda}\left(2^{k} s-2 n+1\right) d s$.

Expand the equation (3.11) at the collocation points, $x_{i}=\frac{2 i-1}{2^{k} M}$, where $i=1,2, \ldots, 2^{k-1} M$, to obtain

$$
\mathbf{Q}_{\hat{\mathbf{m}} \times \hat{\mathbf{m}}}^{\mathbf{g}, \lambda, \alpha, \mathbf{b}}=\mathbf{V}_{\hat{\mathbf{m}} \times \mathbf{1}}^{\lambda, \alpha, \mathbf{b}} \mathbf{B}_{\mathbf{1} \times \hat{\mathbf{m}}},
$$

where $\mathbf{B}_{\mathbf{1} \times \hat{\mathbf{m}}}=\left[g\left(x_{1}\right), g\left(x_{2}\right), \ldots, g\left(x_{\hat{m}}\right)\right], \mathbf{V}_{\hat{\mathbf{m}} \times \mathbf{1}}^{\lambda, \alpha, \mathbf{b}}=\left[V_{1,0}^{\lambda, \alpha, b}, V_{1,1}^{\lambda, \alpha, b}, \ldots, V_{1 M-1}^{\lambda, \alpha, b}\right.$, $\left.V_{2,0}^{\lambda, \alpha, b}, V_{2,1}^{\lambda, \alpha, b}, \ldots, V_{2, M-1}^{\lambda, \alpha, b}, \ldots, V_{2^{k-1}, 0}^{\lambda, \alpha, b}, V_{2^{k-1}, 1}^{\lambda, \alpha, b}, \ldots, V_{2^{k-1}, M-1}^{\lambda, \alpha, b}\right]^{T}$,

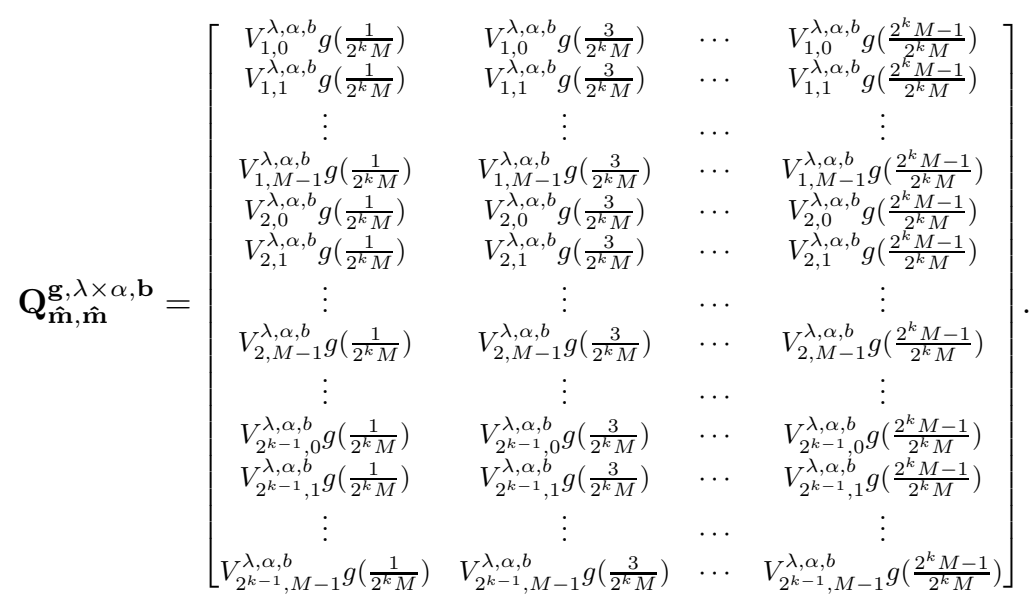


In particular, for $\lambda=7, k=2, M=3, \alpha=1.5, b=1$, and $g(x)=x^{2}$, we have

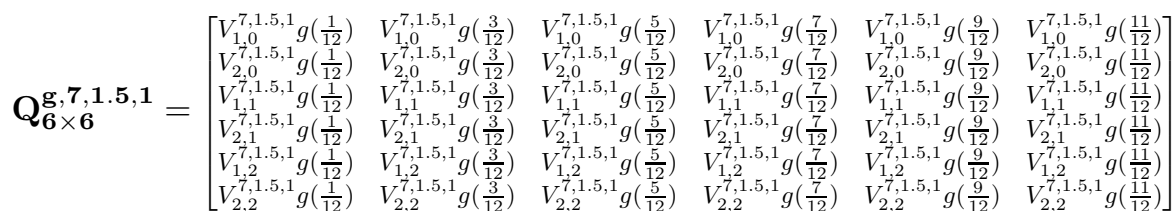

or

$$
\mathbf{Q}_{\mathbf{6} \times \mathbf{6}}^{\mathbf{g}, \mathbf{7 , 1 . 5 , 1}}=\left[\begin{array}{cccccc}
0.0103 & 0.0924 & 0.2566 & 0.5029 & 0.8313 & 1.2419 \\
0.0056 & 0.0505 & 0.1403 & 0.2750 & 0.4547 & 0.6792 \\
-0.0007 & -0.0059 & -0.0165 & -0.0324 & -0.0535 & -0.0799 \\
-0.0013 & -0.0115 & -0.0321 & -0.0629 & -0.1039 & -0.1552 \\
0.0038 & 0.0341 & 0.0949 & 0.1859 & 0.3073 & 0.4591 \\
0.0019 & 0.0174 & 0.0485 & 0.0950 & 0.1570 & 0.2345
\end{array}\right] .
$$

Theorem (Convergence of the Gegenbauer wavelets method). Let $2^{k-1}, M \rightarrow$ $\infty$. Then the series solution (3.4) converges to $f(x)$.

Proof. Take the inner product of $f(x)$ and $\psi_{n, m}^{\lambda}(x)$ with respect to the weight function, given in equations (3.2) and (3.3), we get

$$
a_{n m}^{\lambda}=\left\langle f(x), \psi_{n, m}^{\lambda}(x)\right\rangle=\int_{0}^{1} w_{n, k}(x) f(x) \psi_{n, m}^{\lambda}(x) d x .
$$

We introduce notations, $\hat{p}=2^{k-1}, p=2^{d-1}, \hat{q}=M$ and $q=N$, where $k$ and $d$ denotes the level of resolutions and, $M$ and $N$ represents the order of the Gegenbauer polynomials.

Let $S_{\hat{p}, \hat{q}}^{\lambda}$ be a sequence of partial sums of $a_{i j}^{\lambda} \psi_{i, j}^{\lambda}(x)$, we prove that $S_{\hat{p}, \hat{q}}$ is a Cauchy sequence in Hilbert space $L^{2}[0,1)$ and then we show that $S_{\hat{p}, \hat{q}}^{\lambda}$ converges to $f(x)$, when $\hat{p}, \hat{q} \rightarrow \infty$.

First we show that $S_{\hat{p}, \hat{q}}^{\lambda}$ is a Cauchy sequence. For this purpose, let $S_{p, q}^{\lambda}$ be arbitrary sums of $a_{i j}^{\lambda} \psi_{i, j}^{\lambda}(x)$ with $\hat{p}>p, \hat{q}>q$.

$$
\begin{aligned}
\left\|S_{\hat{p}, \hat{q}}^{\lambda}-S_{p, q}^{\lambda}\right\|^{2} & =\left\|\sum_{i=p+1}^{\hat{p}} \sum_{j=q}^{\hat{q}-1} a_{i j}^{\lambda} \psi_{i, j}^{\lambda}(x)\right\|^{2} \\
& =\left\langle\sum_{i=p+1}^{\hat{p}} \sum_{j=q}^{\hat{q}-1} a_{i j}^{\lambda} \psi_{i, j}^{\lambda}(x), \sum_{r=p+1}^{\hat{p}} \sum_{s=q}^{\hat{q}-1} a_{r s}^{\lambda} \psi_{r, s}^{\lambda}(x)\right\rangle \\
& =\sum_{i=p+1}^{\hat{p}} \sum_{j=q}^{\hat{q}-1} \sum_{r=p+1}^{\hat{p}} \sum_{s=q}^{\hat{q}-1} a_{i j}^{\lambda} \bar{a}_{r s}^{\lambda}\left\langle\psi_{i, j}^{\lambda}(x), \psi_{r, s}^{\lambda}(x)\right\rangle \\
& =\sum_{i=p+1}^{\hat{p}} \sum_{j=q}^{\hat{q}-1}\left|a_{i j}^{\lambda}\right|^{2} .
\end{aligned}
$$



hence

From the Bessel's inequality, we have $\sum_{i=1}^{\infty} \sum_{j=0}^{\infty}\left|a_{i j}^{\lambda}\right|^{2}$ is convergent and

$$
\left\|S_{\hat{p}, \hat{q}}^{\lambda}-S_{p, q}^{\lambda}\right\|^{2} \rightarrow 0 \text { as } \hat{p}, \hat{q}, p, q \rightarrow \infty .
$$

This implies that $S_{\hat{p}, \hat{q}}^{\lambda}$ is a Cauchy sequence and it converges to, say, $y(x) \in$ $L^{2}[0,1)$. We need to show that $y(x)=f(x)$,

$$
\begin{aligned}
\left\langle y(x)-f(x), \psi_{i, j}^{\lambda}(x)\right\rangle & =\left\langle y(x), \psi_{i, j}^{\lambda}(x)\right\rangle-\left\langle f(x), \psi_{i, j}^{\lambda}(x)\right\rangle \\
& =\lim _{\hat{p}, \hat{q} \rightarrow \infty}\left\langle S_{\hat{p}, \hat{q}}^{\lambda}, \psi_{i, j}^{\lambda}(x)\right\rangle-a_{i j}^{\lambda} \\
& =a_{i j}^{\lambda}-a_{i j}^{\lambda} \\
& =0 .
\end{aligned}
$$

Hence $\sum_{i=1}^{\hat{p}} \sum_{j=0}^{\hat{q}-1} a_{i j}^{\lambda} \psi_{i, j}^{\lambda}(x)$ converges to $f(x)$ as $\hat{p}, \hat{q} \rightarrow \infty$.

\section{Implementation and examples}

We describe the algorithm by working out few examples. In this section, we implement the Gegenbauer wavelets method to fractional initial as well as boundary value problems.

\section{Initial value problems}

Example 1. Consider the following Bagley-Torvik equation,

$$
\begin{aligned}
b_{1} D^{2} y(x)+b_{2}^{c} D^{1.5} y(x)+b_{3} y(x) & =f(x), x \geq 0, \\
y(0) & =1, y^{\prime}(0)=1,
\end{aligned}
$$

where $f(x)=b_{3}(x+1)$, and the exact solution [28] is $y(x)=x+1$. Apply the Gegenbauer wavelet method to equation (4.1), we approximate the higher order term by the Gegenbauer wavelets series as

$$
D^{2} y(x)=\sum_{n=1}^{2^{k-1}} \sum_{m=0}^{M-1} a_{n m}^{\lambda} \psi_{n, m}^{\lambda}(x)=\mathbf{a}^{\lambda^{T}} \Psi^{\lambda}(x) .
$$

Lower order derivatives are obtained by integrating (4.2) and use the initial condition

$$
\begin{gathered}
D y(x)=\sum_{n=1}^{2^{k-1}} \sum_{m=0}^{M-1} a_{n m}^{\lambda}\left(I_{0}^{1} \psi_{n, m}^{\lambda}(x)\right)+1=\mathbf{a}^{\lambda^{T}} \mathbf{P}^{\lambda, 1} \Psi^{\lambda}(x)+1, \\
y(x)=\sum_{n=1}^{2^{k-1}} \sum_{m=0}^{M-1} a_{n m}^{\lambda}\left(I_{0}^{2} \psi_{n, m}^{\lambda}(x)\right)+x+1=\mathbf{a}^{\lambda^{T}} \mathbf{P}^{\lambda, 2} \Psi^{\lambda}(x)+x+1, \\
D^{1.5} y(x)=\sum_{n=1}^{2^{k-1}} \sum_{m=0}^{M-1} a_{n m}^{\lambda}\left(I_{0}^{0.5} \psi_{n, m}^{\lambda}(x)\right)=\mathbf{a}^{\lambda^{T}} \mathbf{P}^{\lambda, 0.5} \Psi^{\lambda}(x) .
\end{gathered}
$$


Substitute (4.2), (4.4), and (4.5) in equation (4.1), we get

$$
\begin{aligned}
& \sum_{n=1}^{2^{k-1}} \sum_{m=0}^{M-1}\left(b_{1} a_{n m}^{\lambda} \psi_{n, m}^{\lambda}(x)+b_{2} a_{n m}^{\lambda}\left(I_{0}^{0.5} \psi_{n, m}^{\lambda}(x)\right)+b_{3} a_{n m}^{\lambda}\left(I_{0}^{2} \psi_{n, m}^{\lambda}(x)\right)\right. \\
= & -b_{3}(1+x)+f(x), x \geq 0 .
\end{aligned}
$$

Equation (4.6) at the collocation points, $x_{i}=\frac{2 i-1}{2^{k} M}$, where $i=1,2, \ldots$, $2^{k-1} M$, and in vector notation, takes the following form by using equations (3.5) and (3.9)

$$
\begin{aligned}
& \mathbf{a}^{\lambda^{T}}\left(b_{1} \Psi_{\hat{m} \times \hat{m}}^{\lambda}+b_{2} \mathbf{P}_{\hat{m} \times \hat{m}}^{\lambda, 0.5} \Psi_{\hat{m} \times \hat{m}}^{\lambda}+b_{3} \mathbf{P}_{\hat{m} \times \hat{m}}^{\lambda, 2} \Psi_{\hat{m} \times \hat{m}}^{\lambda}\right)=F \text {, or } \\
& \mathbf{a}^{\lambda^{T}} U_{\hat{m} \times \hat{m}}^{\lambda}=F,
\end{aligned}
$$

where $F=\left\{-b_{3}\left(1+x_{i}\right)+f\left(x_{i}\right)\right\}_{i=1}^{2^{k-1} M}$ and $U_{\hat{m} \times \hat{m}}^{\lambda}=\left(b_{1} \Psi_{\hat{m} \times \hat{m}}^{\lambda}+b_{2} \mathbf{P}_{\hat{m} \times \hat{m}}^{\lambda, 0.5} \Psi_{\hat{m} \times \hat{m}}^{\lambda}\right.$ $\left.+b_{3} \mathbf{P}_{\hat{m} \times \hat{m}}^{\lambda, 2} \Psi_{\hat{m} \times \hat{m}}^{\lambda}\right)$.

Consider $b_{1}=b_{2}=b_{3}=1$ and $\lambda=17.5, k=2, M=4$, we get

$$
\begin{aligned}
& U_{8 \times 8}^{17.5}=\left(\begin{array}{cccccccc}
3.9117 & 4.6393 & 5.1779 & 5.6801 & 2.2699 & 2.0590 & 2.0610 & 2.1288 \\
0 & 0 & 0 & 0 & 3.9117 & 4.6393 & 5.1779 & 5.6801 \\
-17.8455 & -9.2678 & 2.3847 & 15.8417 & 1.0869 & 0.1013 & -0.1984 & -0.3492 \\
0 & 0 & 0 & 0 & -17.8455 & -9.2678 & 2.3847 & 15.8417 \\
57.0389 & 14.3879 & 12.3357 & 65.5851 & 18.9272 & 16.3578 & 16.1898 & 16.6459 \\
0 & 0 & 0 & 0 & 57.0389 & 14.3879 & 12.3357 & 65.5851 \\
-147.2366 & -25.9284 & -21.4612 & 128.2630 & 8.4966 & 0.8268 & -1.4405 & -2.5715 \\
0 & 0 & 0 & 0 & -147.2366 & -25.9284 & -21.4612 & 128.2630
\end{array}\right) \text {, } \\
& F=\left(\begin{array}{llllllll}
0 & 0 & 0 & 0 & 0 & 0 & 0 & 0
\end{array}\right), \mathbf{a}^{17.5^{T}}=\left(\begin{array}{llllllll}
0 & 0 & 0 & 0 & 0 & 0 & 0 & 0
\end{array}\right) .
\end{aligned}
$$

Substitute $\mathbf{a}^{17.5^{T}}$ in equation (4.4) to obtain

$$
y(x)=x+1,
$$

which is the exact solution.

Example 2. Consider the following fractional differential equation with variable coefficients,

$$
\begin{aligned}
& g D^{2} y(x)+b(x)^{c} D^{\alpha} y(x)+c(x) D y(x)+e(x)^{c} D^{\beta} y(x) \\
& +k(x) y(x)=f(x), 0<\beta<1,1<\alpha<2, \\
& y(0)=2, y^{\prime}(0)=0
\end{aligned}
$$

where $g$ is a constant and $f(x)=-g-\frac{b(x)}{\Gamma(3-\alpha)} x^{2-\alpha}-x c(x)-\frac{e(x)}{\Gamma(3-\beta)} x^{2-\beta}+$ $k(x)\left(2-\frac{1}{2} x^{2}\right)$, and the exact solution [13] is $y(x)=2-\frac{1}{2} x^{2}$.

Apply the Gegenbauer wavelets method to equation (4.9), we obtain

$$
\begin{aligned}
& \mathbf{a}^{\lambda^{T}}\left(g \Psi_{\hat{m} \times \hat{m}}^{\lambda}+\mathbf{P}_{\hat{m} \times \hat{m}}^{\lambda, 2-\alpha} \Psi_{\hat{m} \times \hat{m}}^{\lambda} B+\mathbf{P}_{\hat{m} \times \hat{m}}^{\lambda, 1} \Psi_{\hat{m} \times \hat{m}}^{\lambda} C\right. \\
& \left.+\mathbf{P}_{\hat{m} \times \hat{m}}^{\lambda, 2-\beta} \Psi_{\hat{m} \times \hat{m}}^{\lambda} E+\mathbf{P}_{\hat{m} \times \hat{m}}^{\lambda, 2} \Psi_{\hat{m} \times \hat{m}}^{\lambda} K\right)=L,
\end{aligned}
$$

and solution at the collocation points is given by

$$
Y=\mathbf{a}^{\lambda^{T}} \mathbf{P}_{\hat{m} \times \hat{m}}^{\lambda, 2} \Psi_{\hat{m} \times \hat{m}}^{\lambda}+2,
$$


where

$$
\begin{aligned}
L & =\left[f\left(x_{1}\right)-2 k\left(x_{1}\right), f\left(x_{2}\right)-2 k\left(x_{2}\right), \ldots, f\left(x_{\hat{m}}\right)-2 k\left(x_{\hat{m}}\right)\right], \\
Y & =\left[y\left(x_{1}, y\left(x_{2}\right), \ldots, y\left(x_{\hat{m}}\right)\right)\right]
\end{aligned}
$$

and $B, C, E$ and $K$ are the diagonal matrices and are given by

$$
\begin{aligned}
& B=\left[\begin{array}{cccc}
b\left(x_{1}\right) & 0 & \cdots & 0 \\
0 & b\left(x_{2}\right) & \cdots & 0 \\
\vdots & \vdots & \ddots & \vdots \\
0 & 0 & \cdots & b\left(x_{\hat{m}}\right)
\end{array}\right], C=\left[\begin{array}{cccc}
c\left(x_{1}\right) & 0 & \cdots & 0 \\
0 & c\left(x_{2}\right) & \cdots & 0 \\
\vdots & \vdots & \ddots & \vdots \\
0 & 0 & \cdots & c\left(x_{\hat{m}}\right)
\end{array}\right], \\
& E=\left[\begin{array}{cccc}
e\left(x_{1}\right) & 0 & \cdots & 0 \\
0 & e\left(x_{2}\right) & \cdots & 0 \\
\vdots & \vdots & \ddots & \vdots \\
0 & 0 & \cdots & e\left(x_{\hat{m}}\right)
\end{array}\right] \text { and } K=\left[\begin{array}{cccc}
k\left(x_{1}\right) & 0 & \cdots & 0 \\
0 & k\left(x_{2}\right) & \cdots & 0 \\
\vdots & \vdots & \ddots & \vdots \\
0 & 0 & \cdots & k\left(x_{\hat{m}}\right)
\end{array}\right],
\end{aligned}
$$

where $x_{i}=\frac{2 i-1}{2 \hat{m}}, i=1,2, \ldots, \hat{m}$, are collocation points.

We consider $g=1, b(x)=\sqrt{x}, c(x)=e^{x}, e(x)=x^{\frac{1}{4}}$, and $k(x)=x^{\frac{1}{5}}$. We fix $\alpha=1.755, \beta=0.333$ and plot the exact solution and solution by the Gegenbauer wavelets method at $k=5, M=11, \lambda=30$, as shown in Figure 1, along with the absolute error. Table 1 shows that absolute error reduces while increasing $k$ and $M$, as in convergence analysis.
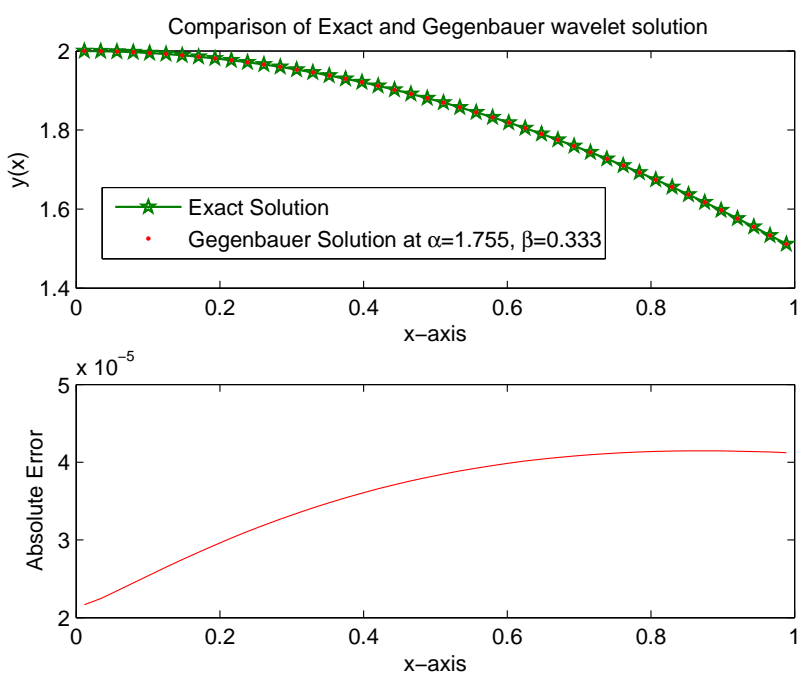

Figure 1. Exact solution and solution by the Gegenbauer wavelet method by taking $k=5, M=11, \lambda=30$, at $\beta=$ 0.333 and $\alpha=1.755$. 
TABLE 1. Absolute error for different values of $k$ and $M$, at $\lambda=7$, and $\alpha=1.8, \beta=0.7$.

\begin{tabular}{lllll}
\hline$x$ & $k=2, M=3$ & $k=4, M=5$ & $k=6, M=7$ & $k=7, M=9$ \\
\hline 0.1 & $1.1894 \times 10^{-3}$ & $0.3059 \times 10^{-4}$ & $0.1174 \times 10^{-5}$ & $0.2017 \times 10^{-6}$ \\
0.2 & $1.2516 \times 10^{-3}$ & $0.3553 \times 10^{-4}$ & $0.1496 \times 10^{-5}$ & $0.2709 \times 10^{-6}$ \\
0.3 & $1.3127 \times 10^{-3}$ & $0.3969 \times 10^{-4}$ & $0.1767 \times 10^{-5}$ & $0.3289 \times 10^{-6}$ \\
0.4 & $1.3649 \times 10^{-3}$ & $0.4304 \times 10^{-4}$ & $0.1989 \times 10^{-5}$ & $0.3765 \times 10^{-6}$ \\
0.5 & $1.4015 \times 10^{-3}$ & $0.4563 \times 10^{-4}$ & $0.2164 \times 10^{-5}$ & $0.4145 \times 10^{-6}$ \\
0.6 & $1.4233 \times 10^{-3}$ & $0.4753 \times 10^{-4}$ & $0.2298 \times 10^{-5}$ & $0.4439 \times 10^{-6}$ \\
0.7 & $1.4333 \times 10^{-3}$ & $0.4883 \times 10^{-4}$ & $0.2396 \times 10^{-5}$ & $0.4656 \times 10^{-6}$ \\
0.8 & $1.4331 \times 10^{-3}$ & $0.4960 \times 10^{-4}$ & $0.2462 \times 10^{-5}$ & $0.4806 \times 10^{-6}$ \\
0.9 & $1.4242 \times 10^{-3}$ & $0.4993 \times 10^{-4}$ & $0.2501 \times 10^{-5}$ & $0.4901 \times 10^{-6}$ \\
1.0 & $1.4079 \times 10^{-3}$ & $0.4991 \times 10^{-4}$ & $0.2517 \times 10^{-5}$ & $0.4948 \times 10^{-6}$ \\
\hline
\end{tabular}

\section{Boundary value problems}

Example 3. Consider the following fractional boundary value problem,

$$
\begin{aligned}
{ }^{c} D^{\alpha} y(x) & ={ }^{c} D^{\beta} y(x)+h(x), 1<\alpha \leq 2,0<\beta \leq 1, \\
y(0) & =0, y(1)=0,
\end{aligned}
$$

where $h(x)=-e^{x-1}-1$, and the exact solution [30] is known at $\alpha=2$ and $\beta=1, y(x)=x\left(1-e^{x-1}\right)$.

Consider the Gegenbauer wavelet approximation of higher order derivative term in Eq. (4.12)

$$
{ }^{c} D^{\alpha} y(x)=\sum_{n=1}^{2^{k-1}} \sum_{m=0}^{M-1} a_{n m}^{\lambda} \psi_{n, m}^{\lambda}(x)
$$

Now to get the Gegenbauer wavelet series for lower order derivative terms we integrate Eq. (4.13) and use the boundary condition, to get

$$
\begin{aligned}
y(x)= & \sum_{n=1}^{2^{k-1}} \sum_{m=0}^{M-1} a_{n m}^{\lambda}\left(I_{0}^{\alpha} \psi_{n, m}^{\lambda}(x)-x\left(\frac{1}{\Gamma(\alpha)} \int_{0}^{1}(1-s)^{\alpha-1} \psi_{n, m}(s) d s\right)\right), \\
{ }^{c} D^{\beta} y(x)= & \sum_{n=1}^{2^{k-1}} \sum_{m=0}^{M-1} a_{n m}^{\lambda}\left(I_{0}^{\alpha-\beta} \psi_{n, m}^{\lambda}(x)\right. \\
& \left.-\frac{\Gamma(2)}{\Gamma(2-\beta)} x^{1-\beta}\left(\frac{1}{\Gamma(\alpha)} \int_{0}^{1}(1-s)^{\alpha-1} \psi_{n, m}(s) d s\right)\right) .
\end{aligned}
$$


Substitute Eqs. (4.13) and (4.14) in equation (4.12), we obtain

$$
\begin{aligned}
& \sum_{n=1}^{2^{k-1}} \sum_{m=0}^{M-1} a_{n m}^{\lambda}\left(\psi_{n, m}^{\lambda}(x)-I_{0}^{\alpha-\beta} \psi_{n, m}^{\lambda}(x)\right. \\
& \left.\quad+\frac{\Gamma(2)}{\Gamma(2-\beta)} x^{1-\beta}\left(\frac{1}{\Gamma(\alpha)} \int_{0}^{1}(1-s)^{\alpha-1} \psi_{n, m}(s) d s\right)\right)=h(x) .
\end{aligned}
$$

Use equations (3.5), (3.9) and (3.12), we get

$$
\mathbf{a}^{\lambda^{T}}\left(\Psi_{\hat{m} \times \hat{m}}^{\lambda}-\mathbf{P}_{\hat{m} \times \hat{m}}^{\lambda, \alpha-\beta} \Psi_{\hat{m} \times \hat{m}}^{\lambda}+\mathbf{Q}_{\hat{\mathbf{m}} \times \hat{\mathbf{m}}}^{\mathbf{g}, \lambda, \alpha, \mathbf{1}}\right)=H,
$$

and solution at the collocation points is given by

$$
Y=\mathbf{a}^{\lambda^{T}} \mathbf{P}_{\hat{m} \times \hat{m}}^{\lambda, \alpha} \Psi_{\hat{m} \times \hat{m}}^{\lambda}-\mathbf{a}^{\lambda^{T}} \mathbf{Q}_{\hat{\mathbf{m}} \times \hat{\mathbf{m}}}^{\mathbf{R}, \lambda, \alpha},
$$

where $H=\left[h\left(x_{1}\right), h\left(x_{2}\right), \ldots, h\left(x_{\hat{m}}\right)\right]$, and $g=\frac{\Gamma(2)}{\Gamma(2-\beta)} x^{1-\beta}, R=x$.

Solve the equation (4.16) for $\mathbf{a}^{\lambda^{T}}$, and substitute it in (4.17) to get $y(x)$, at the collocation points. Choose $\lambda=5, k=10, M=3$, the results obtained by the Gegenbauer wavelet method $y_{G W M}$ is shown in Table 2 along with the absolute error $E_{G W M}$. It shows that Gegenbauer wavelet method provides better results as compared to homotopy perturbation method $y_{H P M}[37]$ and Haar wavelet method $y_{H A A R}[30]$. We can get more accurate results while increasing $k$ or $M$ or both. According to Figure 2, solution by the Gegenbauer wavelet method at different values of $\alpha$ converge to the exact solution, when $\alpha$ approaches to 2 .

TABLE 2. Comparison of the Gegenbauer wavelet method with homotopy perturbation and Haar wavelet method.

\begin{tabular}{lccccc}
\hline \multicolumn{7}{c}{$\begin{array}{c}\lambda=5, k=10, M=3 \\
\alpha=2, \beta=1\end{array}$} \\
\hline$x$ & $y_{H P M}[37]$ & $y_{H A A R}[30]$ & $y_{G W M}$ & $y_{\text {Exact }}$ & $E_{G W M}$ \\
\hline 0.1 & 0.05934820 & 0.05934300 & 0.05934302 & 0.05934303 & $1.58953244 \mathrm{e}-8$ \\
0.2 & 0.11014318 & 0.11013418 & 0.11013419 & 0.11013421 & $1.97211689 \mathrm{e}-8$ \\
0.3 & 0.15103441 & 0.15102438 & 0.15102438 & 0.15102441 & $2.46505651 \mathrm{e}-8$ \\
0.4 & 0.18048329 & 0.18047531 & 0.18047531 & 0.18047535 & $3.08917651 \mathrm{e}-8$ \\
0.5 & 0.19673826 & 0.19673463 & 0.19673463 & 0.19673467 & $3.86865598 \mathrm{e}-8$ \\
0.6 & 0.19780653 & 0.19780792 & 0.19780792 & 0.19780797 & $4.83152341 \mathrm{e}-8$ \\
0.7 & 0.18142196 & 0.18142718 & 0.18142719 & 0.18142725 & $6.01022115 \mathrm{e}-8$ \\
0.8 & 0.14500893 & 0.14501532 & 0.14501532 & 0.14501540 & $7.44224910 \mathrm{e}-8$ \\
0.9 & 0.08564186 & 0.08564623 & 0.08564623 & 0.08564632 & $9.17089691 \mathrm{e}-8$ \\
\hline
\end{tabular}

Example 4. Consider the following fractional boundary value problem with variable coefficients,

$$
\begin{aligned}
& { }^{c} D^{\alpha} y(x)+e(x) D y(x)+b(x)^{c} D^{\beta} y(x) \\
& +c(x) y(x)=f(x), 1<\alpha \leq 2,0<\beta<1, \\
& y(0)=0, y(1)=0,
\end{aligned}
$$




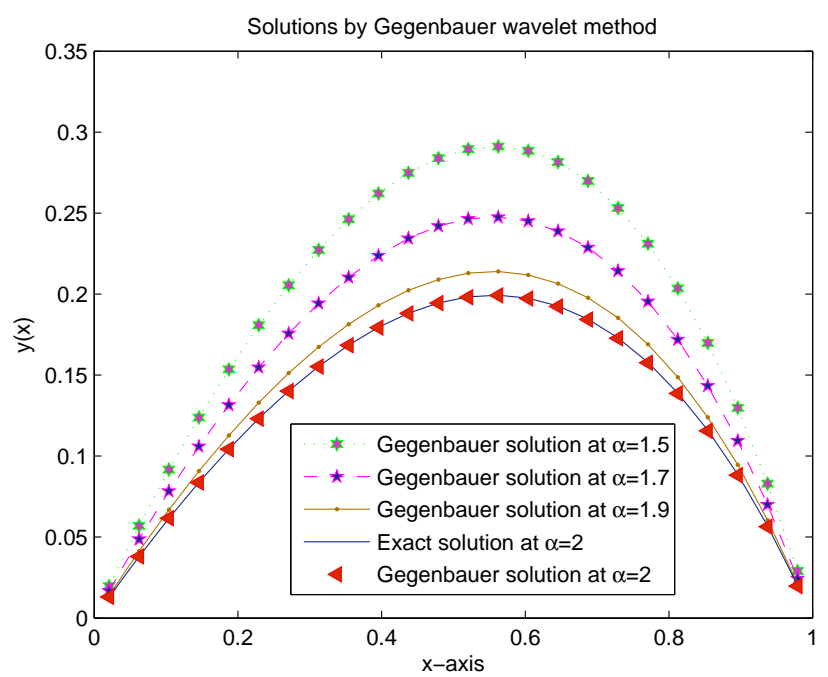

FiguRE 2. Solution by the Gegenbauer wavelet method by taking $k=4, M=3, \lambda=5$, at $\beta=1$ and different $\alpha$.

where $f(x)=\Gamma(\alpha+1)-\frac{\Gamma(8)}{\Gamma(8-\beta)} x^{7-\alpha}+e(x)\left(\alpha x^{\alpha-1}-7 x^{6}\right)+b(x)\left(\frac{\Gamma(\alpha+1)}{\Gamma(\alpha-\beta+1)} x^{\alpha-\beta}-\right.$ $\left.\frac{\Gamma(8)}{\Gamma(8-\beta)} x^{7-\beta}\right)+c(x)\left(x^{\alpha}-x^{7}\right)$, and the exact solution depends on the order of the equation (4.18), $y(x)=x^{\alpha}-x^{7}$.

Apply the Gegenbauer wavelets method to equation (4.18), we get

$$
\begin{aligned}
& \mathbf{a}^{\lambda^{T}}\left(\Psi_{\hat{m} \times \hat{m}}^{\lambda}+\mathbf{P}_{\hat{m} \times \hat{m}}^{\lambda, \alpha-1} \Psi_{\hat{m} \times \hat{m}}^{\lambda} E+\mathbf{P}_{\hat{m} \times \hat{m}}^{\lambda, \alpha-\beta} \Psi_{\hat{m} \times \hat{m}}^{\lambda} B+\mathbf{P}_{\hat{m} \times \hat{m}}^{\lambda, \alpha} \Psi_{\hat{m} \times \hat{m}}^{\lambda} C\right. \\
& \left.-\mathbf{Q}_{\hat{\mathbf{m}} \times \hat{\mathbf{m}}}^{\mathbf{e}, \lambda, \alpha, \mathbf{1}}-\mathbf{Q}_{\hat{\mathbf{m}} \times \hat{\mathbf{m}}}^{\mathbf{g}, \lambda, \alpha, \mathbf{1}}-\mathbf{Q}_{\hat{\mathbf{m} \times \mathbf{m}}}^{\mathbf{h}, \lambda, \alpha, \mathbf{1}}\right)=F,
\end{aligned}
$$

and solution at the collocation points is given by

$$
Y=\mathbf{a}^{\lambda^{T}} \mathbf{P}_{\hat{m} \times \hat{m}}^{\lambda, \alpha} \Psi_{\hat{m} \times \hat{m}}^{\lambda}-\mathbf{a}^{\lambda^{T}} \mathbf{Q}_{\hat{\mathbf{m}} \times \hat{\mathbf{m}}}^{\mathbf{R}, \lambda, \alpha, \mathbf{1}}
$$

where $F=\left[f\left(x_{1}\right), f\left(x_{2}\right), \ldots, f\left(x_{\hat{m}}\right)\right], Y=\left[y\left(x_{1}, y\left(x_{2}\right), \ldots, y\left(x_{\hat{m}}\right)\right)\right], e=e(x)$, $g=b(x)\left(\frac{\Gamma(2)}{\Gamma(2-\beta)} x^{1-\beta}\right), h=x c(x), R=x$, and $E, B$ and $C$ are the diagonal matrices and are given by

$$
E=\left[\begin{array}{cccc}
e\left(x_{1}\right) & 0 & \cdots & 0 \\
0 & e\left(x_{2}\right) & \cdots & 0 \\
\vdots & \vdots & \ddots & \vdots \\
0 & 0 & \cdots & e\left(x_{\hat{m}}\right)
\end{array}\right], B=\left[\begin{array}{cccc}
b\left(x_{1}\right) & 0 & \cdots & 0 \\
0 & b\left(x_{2}\right) & \cdots & 0 \\
\vdots & \vdots & \ddots & \vdots \\
0 & 0 & \cdots & b\left(x_{\hat{m}}\right)
\end{array}\right] \text { and }
$$




$$
C=\left[\begin{array}{cccc}
c\left(x_{1}\right) & 0 & \cdots & 0 \\
0 & c\left(x_{2}\right) & \cdots & 0 \\
\vdots & \vdots & \ddots & \vdots \\
0 & 0 & \cdots & c\left(x_{\hat{m}}\right)
\end{array}\right],
$$

where $x_{i}=\frac{2 i-1}{2 \hat{m}}, i=1,2, \ldots, \hat{m}$, are collocation points.

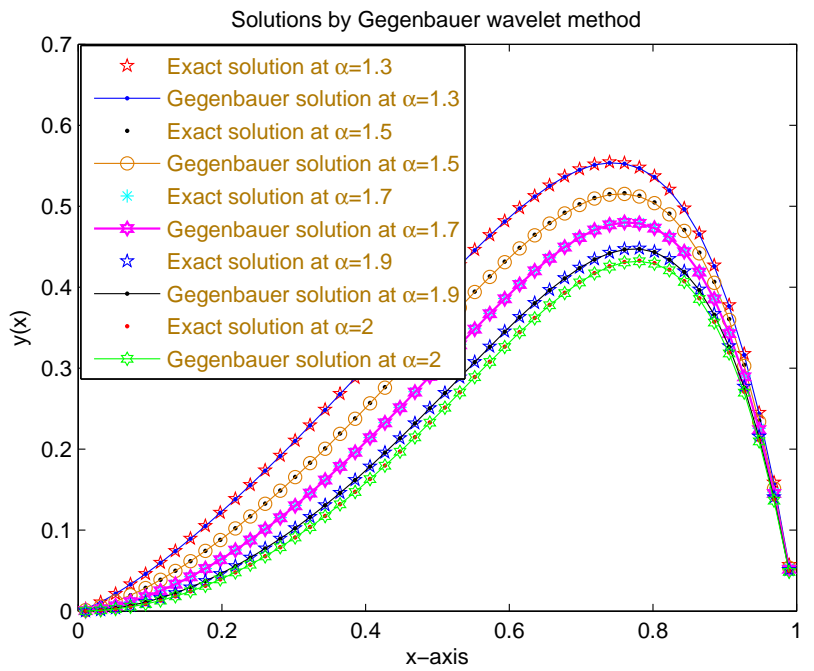

Figure 3. Solution by the Gegenbauer wavelet method by taking $k=5, M=3, \lambda=15$, at $\beta=0.5$ and different $\alpha$.

We consider $e(x)=\sin (x), b(x)=e^{x}, c(x)=x^{2}$ and fix the parameters $k=5, M=3, \lambda=15$. Exact solutions and solutions by Gegenbauer wavelet method agrees at different values of $\alpha$, and $\beta=0.5$, as shown in Figure 3 .

Example 5. Consider the fractional boundary value problem with nonzero boundary conditions,

$$
\begin{aligned}
& { }^{c} D^{\alpha} y(x)+e^{x}{ }^{c} D^{\beta} y(x)+\sin (x) y(x)=f(x), 1<\alpha \leq 2,0<\beta \leq 1, \\
& y(0)=1, y(1)=2,
\end{aligned}
$$

where $f(x)=\frac{\Gamma(\alpha+\beta+1)}{\Gamma(\beta+1)} x^{\beta}+e^{x} \frac{\Gamma(\alpha+\beta+1)}{\Gamma(\alpha+1)} x^{\alpha}+\sin (x)\left(x^{\alpha+\beta}+1\right)$, and the exact solution, $y(x)=x^{\alpha+\beta}+1$, depends on the order of the derivatives appearing in equation (4.21). Let $h(x):=-\frac{\Gamma(2)}{\Gamma(2-\beta)} x^{1-\beta} e^{x}-\sin (x)(1+x)$. Apply the Gegenbauer wavelets method to equation (4.21), we get

(4.22) $\mathbf{a}^{\lambda^{T}}\left(\Psi_{\hat{m} \times \hat{m}}^{\lambda}+\mathbf{P}_{\hat{m} \times \hat{m}}^{\lambda, \alpha-\beta} \Psi_{\hat{m} \times \hat{m}}^{\lambda} A+\mathbf{P}_{\hat{m} \times \hat{m}}^{\lambda, \alpha} \Psi_{\hat{m} \times \hat{m}}^{\lambda} B-\mathbf{Q}_{\hat{\mathbf{m}} \times \hat{\mathbf{m}}}^{\mathbf{e}, \lambda, \alpha, \mathbf{1}}-\mathbf{Q}_{\hat{\mathbf{m}} \times \hat{\mathbf{m}}}^{\mathbf{g}, \lambda, \alpha, \mathbf{1}}\right)=H$, 
and solution at the collocation points is given by

$$
Y=\mathbf{a}^{\lambda^{T}} \mathbf{P}_{\hat{m} \times \hat{m}}^{\lambda, \alpha} \Psi_{\hat{m} \times \hat{m}}^{\lambda}-\mathbf{a}^{\lambda^{T}} \mathbf{Q}_{\hat{\mathbf{m}} \times \hat{\mathbf{m}}}^{\mathbf{R}, \lambda, \alpha, \mathbf{1}}+Z,
$$

where $H=\left[f\left(x_{1}\right)+h\left(x_{1}\right), f\left(x_{2}\right)+h\left(x_{2}\right), \ldots, f\left(x_{\hat{m}}\right)+h\left(x_{\hat{m}}\right)\right], Z=\left[x_{1}+1, x_{2}+\right.$ $\left.1, \ldots, x_{\hat{m}}+1\right], Y=\left[y\left(x_{1}\right), y\left(x_{2}\right), \ldots, y\left(x_{\hat{m}}\right)\right], e=x \sin (x), g=e^{x}\left(\frac{\Gamma(2)}{\Gamma(2-\beta)} x^{1-\beta}\right)$, $R=x$, and $A, B$ are the diagonal matrices and are given by

$$
A=\left[\begin{array}{cccc}
e^{x_{1}} & 0 & \cdots & 0 \\
0 & e^{x_{2}} & \cdots & 0 \\
\vdots & \vdots & \ddots & \vdots \\
0 & 0 & \cdots & e^{x_{\hat{m}}}
\end{array}\right], B=\left[\begin{array}{cccc}
\sin \left(x_{1}\right) & 0 & \cdots & 0 \\
0 & \sin \left(x_{2}\right) & \cdots & 0 \\
\vdots & \vdots & \ddots & \vdots \\
0 & 0 & \cdots & \sin \left(x_{\hat{m}}\right)
\end{array}\right]
$$

where $x_{i}=\frac{2 i-1}{2 \hat{m}}, i=1,2, \ldots, \hat{m}$, are collocation points.

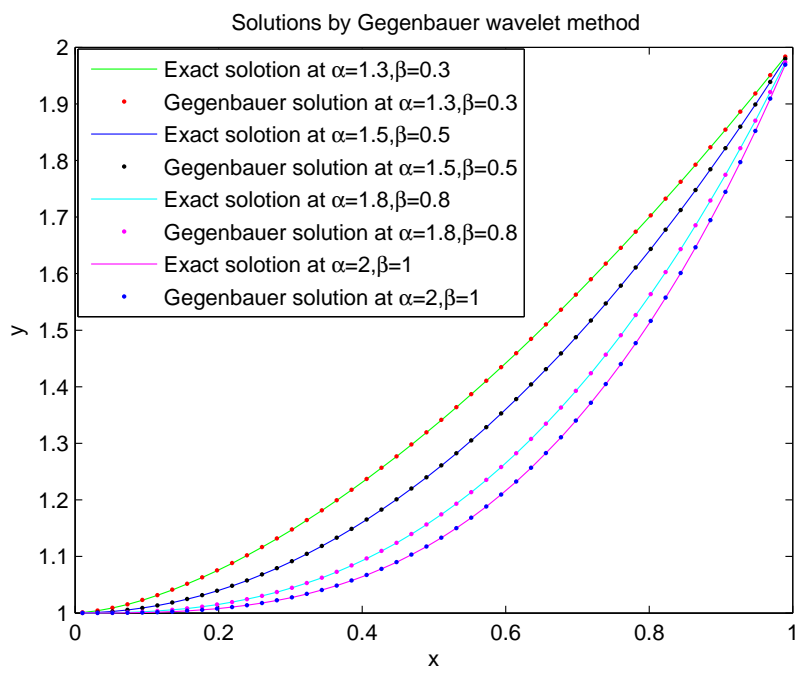

Figure 4. Solution by the Gegenbauer wavelet method by taking $k=5, M=3, \lambda=3$, at different $\alpha$ and $\beta$.

Exact solutions and solutions by Gegenbauer wavelet method at $k=5$, $M=3$ and $\lambda=3$, agrees at different $\alpha$ and $\beta$, as shown in Figure 4 .

\section{Gegenbauer wavelets quasilinearization method}

In this section we focus on nonlinear differential equations. To handle the nonlinearities in equation we use quasilinearization technique, then we solve the linearised problem by Gegenbauer wavelets method. 


\section{Convergence}

\section{Convergence of quasilinearization technique}

Consider the nonlinear second order differential equation

$$
y^{\prime \prime}(x)=f(y), \quad y(0)=y(b)=0 .
$$

Application of quasilinearization technique to equation (5.1) yields

$$
y_{r+1}^{\prime \prime}(x)=f\left(y_{r}\right)+\left(y_{r+1}-y_{r}\right) f^{\prime}\left(y_{r}\right), \quad y_{r+1}(0)=y_{r+1}(b)=0 .
$$

Let $y_{0}(x)$ be some initial approximation. Each function $y_{r+1}(x)$ is a solution of a linear equation (5.2), where $y_{r}$ is always considered known and is obtained from the previous iteration.

According to [6] and letting

$$
\max _{y}\left(|f(y)|,\left|f^{\prime}(y)\right|\right)=m<\infty \text { and } k=\max _{u}\left|f^{\prime \prime}(u)\right|,
$$

we have

$$
\max _{x}\left|y_{r+1}-y_{r}\right| \leq \frac{b^{2} \frac{k}{8}}{1-\frac{b^{2} m}{4}}\left(\max _{x}\left(\left|y_{r}-y_{r-1}\right|\right)^{2} .\right.
$$

This shows that quasilinearization technique has quadratic convergence, if there is convergence at all.

\section{Convergence of Gegenbauer wavelet quasilinearization technique}

According to the convergence analysis of Gegenbauer wavelets method, as derived in Section 3, we have

$$
\sum_{i=1}^{\hat{p}} \sum_{j=0}^{\hat{q}-1} a_{i j}^{\lambda} \psi_{i, j}^{\lambda}(x) \text { converges to } y_{r+1}(x) \text { as } \hat{p}, \hat{q} \rightarrow \infty,
$$

where $\hat{p}=2^{k-1}$ and $\hat{q}=M$. Since $y_{r+1}(x)$ is obtained at $(r+1)$ th iteration of quasilinearization technique, according to convergence analysis of quasilinearization technique (5.3) which states that $y_{r+1}(x)$ converges to $y(x)$ as $r$ approaches to infinity.

This implies that solution by Gegenbauer wavelet quasilinearization technique, $y_{r+1}(x)$, converges to $y(x)$ as $k, M$ and $r$ approaches to $\infty$.

We describe the procedure of implementation of Gegenbauer wavelet quasilinearization technique for both nonlinear initial and boundary value problems.

\subsection{Initial value problems}

Consider the following nonlinear initial value problem

$$
\begin{aligned}
{ }_{c} D^{\alpha} y & =f\left(x, y,{ }_{c} D^{\beta} y, y^{\prime}\right), 0 \leq x \leq \eta, 1<\alpha<2,0<\beta<1, \\
y(0) & =A, y^{\prime}(0)=B
\end{aligned}
$$


where $A$ and $B$ are constants. Apply the quasilinearization technique to equation (5.4), we get

$$
\begin{aligned}
& { }_{c} D^{\alpha} y_{r+1}-f_{y_{r}^{\prime}}\left(x, y_{r},{ }_{c} D^{\beta} y_{r}, y_{r}^{\prime}\right) y_{r+1}^{\prime}-f_{y_{r}^{\beta}}\left(x, y_{r},{ }_{c} D^{\beta} y_{r}, y_{r}^{\prime}\right) y_{r+1}^{\beta} \\
& -f_{y_{r}}\left(x, y_{r},{ }_{c} D^{\beta} y_{r}, y_{r}^{\prime}\right) y_{r+1} \\
= & \left.f\left(x, y_{r},{ }_{c} D^{\beta} y_{r}, y_{r}^{\prime}\right)\right)-y_{r} f_{y_{r}}\left(x, y_{r},{ }_{c} D^{\beta} y_{r}, y_{r}^{\prime}\right)-y_{r}^{\beta} f_{y_{r}^{\beta}}\left(x, y_{r},{ }_{c} D^{\beta} y_{r}, y_{r}^{\prime}\right) \\
& -y_{r}^{\prime} f_{y_{r}^{\prime}}\left(x, y_{r},{ }_{c} D^{\beta} y_{r}, y_{r}^{\prime}\right), y_{r+1}(0)=A, y_{r+1}^{\prime}(0)=B, r \geq 0,
\end{aligned}
$$

where $\frac{\partial f}{\partial y_{r}}=f_{y_{r}}\left(x, y_{r},{ }_{c} D^{\beta} y_{r}, y_{r}^{\prime}\right), \frac{\partial f}{\partial y_{r}^{\prime}}=f_{y_{r}^{\prime}}\left(x, y_{r},{ }_{c} D^{\beta} y_{r}, y_{r}^{\prime}\right)$ and $\frac{\partial f}{\partial y_{r}^{\beta}}=f_{y_{r}^{\beta}}(x$, $\left.y_{r}, D^{\beta} y_{r}, y_{r}^{\prime}\right)$.

Implement the Gegenbauer wavelet method to the series of differential equation (5.5). Approximate the higher order derivative term in Gegenbauer wavelet series as

$$
{ }_{c} D^{\alpha} y_{r+1}=\sum_{n=1}^{2^{k-1}} \sum_{m=0}^{M-1} a_{n m}^{\lambda, r+1} \psi_{n, m}^{\lambda}(x) .
$$

Integrate the equation (5.6) and use the initial conditions, to obtain

$$
y_{r+1}=\sum_{n=1}^{2^{k-1}} \sum_{m=0}^{M-1} a_{n m}^{\lambda, r+1}\left(I_{0}^{\alpha} \psi_{n, m}^{\lambda}(x)\right)+B x+A .
$$

Differentiation of equation (5.7) implies

$$
\begin{aligned}
y_{r+1}^{\prime} & =\sum_{n=1}^{2^{k-1}} \sum_{m=0}^{M-1} a_{n m}^{\lambda, r+1}\left(I_{0}^{\alpha-1} \psi_{n, m}^{\lambda}(x)\right)+B \\
{ }_{c} D^{\beta} y_{r+1} & =\sum_{n=1}^{2^{k-1}} \sum_{m=0}^{M-1} a_{n m}^{\lambda, r+1}\left(I_{0}^{\alpha-\beta} \psi_{n, m}^{\lambda}(x)\right)+B \frac{x^{1-\beta}}{\Gamma(2-\beta)} .
\end{aligned}
$$

Let

$$
\begin{aligned}
g_{r}\left(x, y_{r},{ }_{c} D^{\beta} y_{r}, y_{r}^{\prime}\right)= & f\left(x, y_{r},{ }_{c} D^{\beta} y_{r}, y_{r}^{\prime}\right)-y_{r} f_{y_{r}}\left(x, y_{r},{ }_{c} D^{\beta} y_{r}, y_{r}^{\prime}\right) \\
& -y_{r}^{\beta} f_{y_{r}^{\beta}}\left(x, y_{r},{ }_{c} D^{\beta} y_{r}, y_{r}^{\prime}\right)-y_{r}^{\prime} f_{y_{r}^{\prime}}\left(x, y_{r},{ }_{c} D^{\beta} y_{r}, y_{r}^{\prime}\right)
\end{aligned}
$$

and use equations (5.6), (5.7) and (5.8) in equation (5.5) to obtain

$$
\begin{aligned}
& \quad \sum_{n=1}^{2^{k-1}} \sum_{m=0}^{M-1} a_{n m}^{\lambda, r+1}\left[\psi_{n, m}^{\lambda}(x)-f_{y_{r}^{\prime}}\left(x, y_{r},{ }_{c} D^{\beta} y_{r}, y_{r}^{\prime}\right)\left(I_{0}^{\alpha-1} \psi_{n, m}^{\lambda}(x)\right)\right. \\
& \left.\quad-f_{y_{r}^{\beta}}\left(x, y_{r},{ }_{c} D^{\beta} y_{r}, y_{r}^{\prime}\right)\left(I_{0}^{\alpha-\beta} \psi_{n, m}^{\lambda}(x)\right)-f_{y_{r}}\left(x, y_{r},{ }_{c} D^{\beta} y_{r}, y_{r}^{\prime}\right)\left(I_{0}^{\alpha} \psi_{n, m}^{\lambda}(x)\right)\right] \\
& =g_{r}\left(x, y_{r},{ }_{c} D^{\beta} y_{r}, y_{r}^{\prime}\right)+B f_{y_{r}^{\prime}}\left(x, y_{r},{ }_{c} D^{\beta} y_{r}, y_{r}^{\prime}\right) \\
& \quad+B f_{y_{r}^{\beta}}\left(x, y_{r}, c\right. \\
& \left.\quad D^{\beta} y_{r}, y_{r}^{\prime}\right) \frac{x^{1-\beta}}{\Gamma(2-\beta)}+(B x+A) f_{y_{r}}\left(x, y_{r},{ }_{c} D^{\beta} y_{r}, y_{r}^{\prime}\right) .
\end{aligned}
$$


Let

$$
\begin{aligned}
G_{r}\left(x, y_{r},{ }_{c} D^{\beta} y_{r}, y_{r}^{\prime}\right)= & g_{r}\left(x, y_{r}, c D^{\beta} y_{r}, y_{r}^{\prime}\right)+B f_{y_{r}^{\prime}}\left(x, y_{r},{ }_{c} D^{\beta} y_{r}, y_{r}^{\prime}\right) \\
& +B f_{y_{r}^{\beta}}\left(x, y_{r},{ }_{c} D^{\beta} y_{r}, y_{r}^{\prime}\right) \frac{x^{1-\beta}}{\Gamma(2-\beta)} \\
& +(B x+A) f_{y_{r}}\left(x, y_{r}, c D^{\beta} y_{r}, y_{r}^{\prime}\right) .
\end{aligned}
$$

Equation (5.9) at the collocation points $x_{c}(j)=\eta \frac{2 j-1}{2^{k} M}, j=1,2, \ldots, \hat{m}$, and in vector notation, takes the following form by using equations (3.5) and (3.9)

$$
\begin{gathered}
\mathbf{a}^{\lambda, r+1^{T}}\left(\Psi_{\hat{m} \times \hat{m}}^{\lambda}-\mathbf{F}_{\mathbf{r}}^{\prime} \mathbf{P}_{\hat{m} \times \hat{m}}^{\lambda, \alpha-1} \Psi_{\hat{m} \times \hat{m}}^{\lambda}-\mathbf{F}_{\mathbf{r}}^{\beta} \mathbf{P}_{\hat{m} \times \hat{m}}^{\lambda, \alpha-\beta} \Psi_{\hat{m} \times \hat{m}}^{\lambda}\right. \\
\left.-\mathbf{F}_{\mathbf{r}} \mathbf{P}_{\hat{m} \times \hat{m}}^{\lambda, \alpha} \Psi_{\hat{m} \times \hat{m}}^{\lambda}\right)=\mathbf{K}^{r},
\end{gathered}
$$

where $\mathbf{a}^{\lambda, r+1}=\left[a_{1}^{\lambda, r+1}, a_{2}^{\lambda, r+1}, \ldots, a_{\hat{m}}^{\lambda, r+1}\right]$ is an unknown vector,

$$
\mathbf{K}^{r}=\left[\left.\left.\left.G_{r}\right|_{x_{c}(1)} G_{r}\right|_{x_{c}(2)} \cdots G_{r}\right|_{x_{c}(\hat{m})}\right]
$$

is known vector and $\mathbf{F}_{\mathbf{r}}, \mathbf{F}_{\mathbf{r}}^{\prime}$ and $\mathbf{F}_{\mathbf{r}}^{\beta}$ are the diagonal matrices and are given by

$$
\begin{aligned}
\mathbf{F}_{\mathbf{r}}= & {\left[\begin{array}{cccc}
\left.f_{y_{r}}\right|_{x_{c}(1)} & 0 & \cdots & 0 \\
0 & \left.f_{y_{r}}\right|_{x_{c}(2)} & \cdots & 0 \\
\vdots & \vdots & \ddots & \vdots \\
0 & 0 & \cdots & \left.f_{y_{r}}\right|_{x_{c}(\hat{m})}
\end{array}\right], } \\
\mathbf{F}_{\mathbf{r}}^{\prime} & {\left[\begin{array}{cccc}
\left.f_{y_{r}^{\prime}}\right|_{x_{c}(1)} & 0 & \cdots & 0 \\
0 & \left.f_{y_{r}^{\prime}}\right|_{x_{c}(2)} & \cdots & 0 \\
\vdots & \vdots & \ddots & \vdots \\
0 & 0 & \cdots & \left.f_{y_{r}^{\prime}}\right|_{x_{c}(\hat{m})}
\end{array}\right], \text { and } } \\
\mathbf{F}_{\mathbf{r}}^{\beta}= & {\left[\begin{array}{cccc}
\left.f_{y_{r}^{\beta}}\right|_{x_{c}(1)} & 0 & \cdots & 0 \\
0 & \left.f_{y_{r}^{\beta}}\right|_{x_{c}(2)} & \cdots & 0 \\
\vdots & \vdots & \ddots & \vdots \\
0 & 0 & \cdots & \left.f_{y_{r}^{\beta}}\right|_{x_{c}(\hat{m})}
\end{array}\right] . }
\end{aligned}
$$

The notation $\left.f_{y_{r}}\right|_{x_{c}(j)}$ is used to represent evaluation of $f_{y_{r}}$ at $x=x_{c}(j)$. Gegenbauer coefficients, $\mathbf{a}^{\lambda, r+1}=\left[a_{1}^{\lambda, r+1}, a_{2}^{\lambda, r+1}, \ldots, a_{\hat{m}}^{\lambda, r+1}\right]$ can be obtained from equation (5.10) for each $r \geq 0$ and use these in equations (5.6), (5.7) and (5.8) to get the approximate values of ${ }_{c} D^{\alpha} y_{r+1}, y_{r+1}, y_{r+1}^{\prime}$ and ${ }_{c} D^{\beta} y_{r+1}$ at the collocation points, $x_{c}(j), j=1,2, \ldots, \hat{m}$. In particular, for $r=0$ we get a linear differential equation in $y_{1}(x)$, from equation (5.5), and use equation (5.10) to obtain $\mathbf{a}^{\lambda, 1}=\left[a_{1}^{\lambda, 1}, a_{2}^{\lambda, 1}, \ldots, a_{\hat{m}}^{\lambda, 1}\right]$ which are used in (5.6), (5.7) and (5.8) to get the approximate values of ${ }_{c} D^{\alpha} y_{1}, y_{1}, y_{1}^{\prime}$ and ${ }_{c} D^{\beta} y_{1}$ at the collocation points. Similarly, for $r=1$ we obtain $y_{2}(x)$ and so on. In this way, we obtain a sequence of approximations $y_{1}(x), y_{2}(x), \ldots$. 
Test problem: Consider the following $\alpha^{\text {th }}$ order fractional Van der Pol oscillator problem

(5.11)

${ }^{c} D^{\alpha} y(x)+y^{\prime}(x)+y(x)+y^{2}(x) y^{\prime}(x)=2 \cos (x)-\cos ^{3}(x), 1<\alpha \leq 2, x \geq 0$, subject to the initial conditions

$$
y(0)=0, y^{\prime}(0)=1 .
$$

The exact solution, when $\alpha=2$, is given by $y(x)=\sin (x)$.
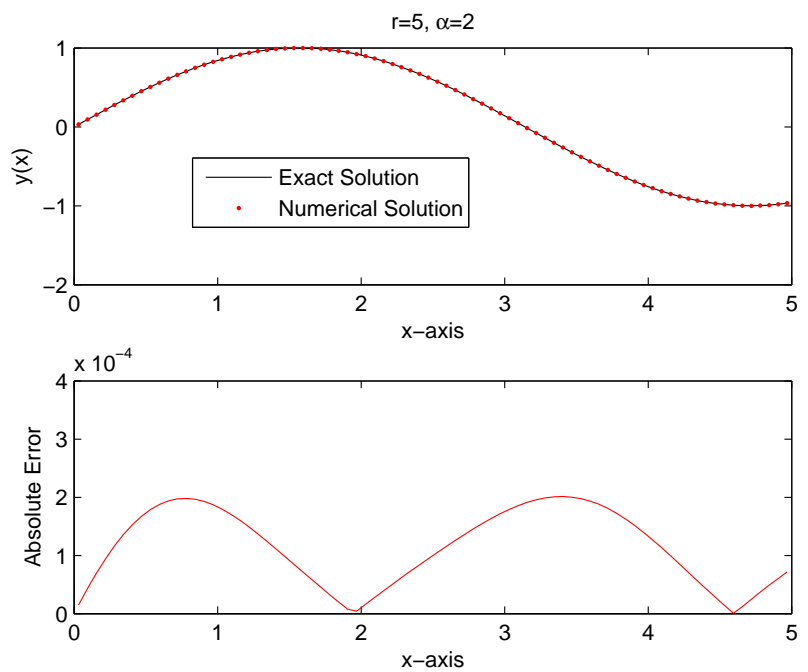

FIGURE 5. Solution by the Gegenbauer wavelet quasilinearization method by taking $k=5, M=5, \lambda=7$, at $\alpha=2$ and $r=5$.

Apply the Gegenbauer wavelet quasilinearization technique to equation (5.11), we get Figures 5 and 6 . Figure 5 shows the comparison of solution by Gegenbauer wavelet quasilinearization technique with exact solution while fixing order of differential equation (5.11), $\alpha=2$, level of resolution, $k=5$, and order of Gegenbauer polynomials, $M=5$, with $\lambda=7$.

Figure 6 indicates the solutions by Gegenbauer wavelet quasilinearization technique at different values of $\alpha^{\prime} s$ by using $k=4, M=7, \lambda=12$ and $r=5$.

\subsection{Boundary value problems}

Consider the fractional nonlinear boundary value problem

$$
\begin{aligned}
{ }_{c} D^{\alpha} y & =f\left(x, y,{ }_{c} D^{\beta} y, y^{\prime}\right), 0 \leq x \leq \eta, 1<\alpha<2,0<\beta<1, \\
y(0) & =\gamma_{1}, y(\eta)=\gamma_{2} .
\end{aligned}
$$




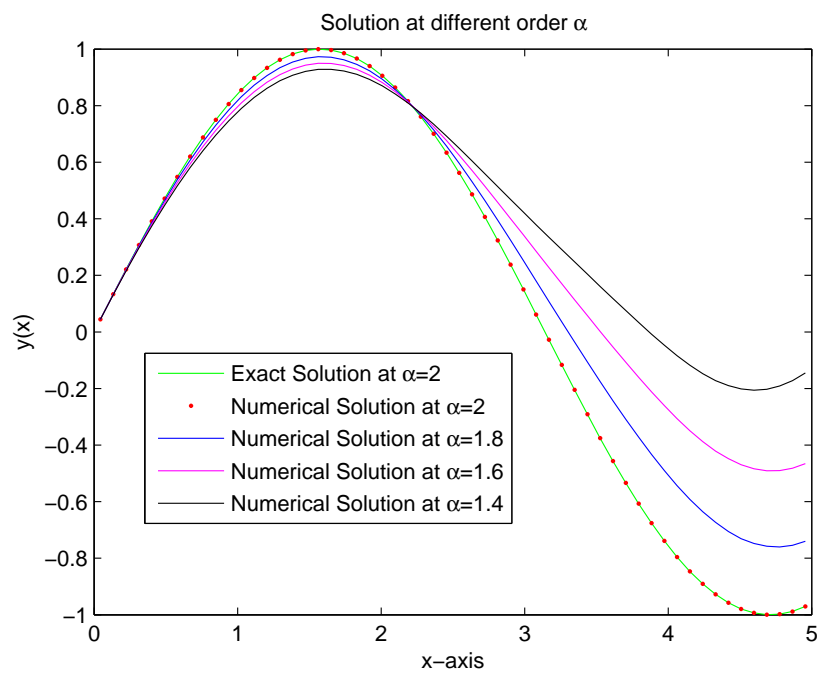

FiguRE 6 . Solution by the Gegenbauer wavelet quasilinearization method by taking $k=4, M=7, \lambda=12$, at different values of $\alpha$ and $r=5$.

Implement the quasilinearization technique to equation (5.12), we get (5.13)

$$
\begin{aligned}
& \left.\left.{ }_{c} D^{\alpha} y_{r+1}-f_{y_{r}^{\prime}}\left(x, y_{r},{ }_{c} D^{\beta} y_{r}, y_{r}^{\prime}\right)\right) y_{r+1}^{\prime}-f_{y_{r}^{\beta}}\left(x, y_{r},{ }_{c} D^{\beta} y_{r}, y_{r}^{\prime}\right)\right) y_{r+1}^{\beta} \\
& \left.-f_{y_{r}}\left(x, y_{r},{ }_{c} D^{\beta} y_{r}, y_{r}^{\prime}\right)\right) y_{r+1} \\
= & \left.\left.\left.f\left(x, y_{r},{ }_{c} D^{\beta} y_{r}, y_{r}^{\prime}\right)\right)-y_{r} f_{y_{r}}\left(x, y_{r},{ }_{c} D^{\beta} y_{r}, y_{r}^{\prime}\right)\right)-y_{r}^{\beta} f_{y_{r}^{\beta}}\left(x, y_{r},{ }_{c} D^{\beta} y_{r}, y_{r}^{\prime}\right)\right) \\
& \left.-y_{r}^{\prime} f_{y_{r}^{\prime}}\left(x, y_{r},{ }_{c} D^{\beta} y_{r}, y_{r}^{\prime}\right)\right), y_{r+1}(0)=\gamma_{1}, y_{r+1}(\eta)=\gamma_{2}, r \geq 0 .
\end{aligned}
$$

Now apply the Gegenbauer wavelet method to the sequence of differential equation (5.13)

$$
{ }_{c} D^{\alpha} y_{r+1}=\sum_{n=1}^{2^{k-1}} \sum_{m=0}^{M-1} a_{n m}^{\lambda, r+1} \psi_{n, m}^{\lambda}(x) .
$$

Integrate the equation (5.14) and use the boundary conditions to obtain

$$
\begin{aligned}
y_{r+1}= & \sum_{n=1}^{2^{k-1}} \sum_{m=0}^{M-1} a_{n m}^{\lambda, r+1}\left(I_{0}^{\alpha} \psi_{n, m}^{\lambda}(x)\right) \\
& +\frac{x}{\eta}\left(\gamma_{2}-\gamma_{1}-\sum_{n=1}^{2^{k-1}} \sum_{m=0}^{M-1} a_{n m}^{\lambda, r+1}\left(\frac{1}{\Gamma(\alpha)} \int_{0}^{\eta}(\eta-s)^{\alpha-1} \psi_{n, m}(s) d s\right)\right)+\gamma_{1} .
\end{aligned}
$$


First and $\beta$ order derivative of $y_{r+1}$ can be obtained from equation (5.15) as $(5.16)$

$$
\begin{aligned}
y_{r+1}^{\prime}= & \sum_{n=1}^{2^{k-1}} \sum_{m=0}^{M-1} a_{n m}^{\lambda, r+1}\left(I_{0}^{\alpha-1} \psi_{n, m}^{\lambda}(x)\right) \\
& +\frac{1}{\eta}\left(\gamma_{2}-\gamma_{1}-\sum_{n=1}^{2^{k-1}} \sum_{m=0}^{M-1} a_{n m}^{\lambda, r+1}\left(\frac{1}{\Gamma(\alpha)} \int_{0}^{\eta}(\eta-s)^{\alpha-1} \psi_{n, m}(s) d s\right)\right), \\
{ }_{c} D^{\beta} y_{r+1}= & \sum_{n=1}^{2^{k-1}} \sum_{m=0}^{M-1} a_{n m}^{\lambda, r+1}\left(I_{0}^{\alpha-\beta} \psi_{n, m}^{\lambda}(x)\right)+\frac{x^{1-\beta}}{\eta \Gamma(2-\beta)}\left(\gamma_{2}-\gamma_{1}\right. \\
& \left.-\sum_{n=1}^{2^{k-1}} \sum_{m=0}^{M-1} a_{n m}^{\lambda, r+1}\left(\frac{1}{\Gamma(\alpha)} \int_{0}^{\eta}(\eta-s)^{\alpha-1} \psi_{n, m}(s) d s\right)\right)+\gamma_{1} .
\end{aligned}
$$

Use equations (5.14), (5.15) and (5.16) in equation (5.13) to obtain (5.17)

$$
\begin{aligned}
& \sum_{n=1}^{2^{k-1}} \sum_{m=0}^{M-1} a_{n m}^{\lambda, r+1}\left[\psi_{n, m}^{\lambda}(x)-f_{y_{r}^{\prime}}\left(x, y_{r}, c D^{\beta} y_{r}, y_{r}^{\prime}\right)\left(I_{x}^{\alpha-1} \psi_{n, m}^{\lambda}(x)\right)\right. \\
& -f_{y_{r}^{\beta}}\left(x, y_{r},{ }_{c} D^{\beta} y_{r}, y_{r}^{\prime}\right)\left(I_{0}^{\alpha-\beta} \psi_{n, m}^{\lambda}(x)\right)-f_{y_{r}}\left(x, y_{r}, c D^{\beta} y_{r}, y_{r}^{\prime}\right)\left(I_{0}^{\alpha} \psi_{n, m}^{\lambda}(x)\right) \\
& \left.+g_{r}\left(x, y_{r}, D^{\beta} y_{r}, y_{r}^{\prime}\right)\left(\frac{1}{\Gamma(\alpha)} \int_{0}^{\eta}(\eta-s)^{\alpha-1} \psi_{n, m}(s) d s\right)\right] \\
& =Q_{r}\left(x, y_{r},{ }_{c} D^{\beta} y_{r}, y_{r}^{\prime}\right) \text {, }
\end{aligned}
$$

where

$$
\begin{aligned}
& Q_{r}\left(x, y_{r},{ }_{c} D^{\beta} y_{r}, y_{r}^{\prime}\right) \\
= & f\left(x, y_{r},{ }_{c} D^{\beta} y_{r}, y_{r}^{\prime}\right)-y_{r} f_{y_{r}}\left(x, y_{r},{ }_{c} D^{\beta} y_{r}, y_{r}^{\prime}\right)-y_{r}^{\beta} f_{y_{r}^{\beta}}\left(x, y_{r},{ }_{c} D^{\beta} y_{r}, y_{r}^{\prime}\right) \\
& -y_{r}^{\prime} f_{y_{r}^{\prime}}\left(x, y_{r},{ }_{c} D^{\beta} y_{r}, y_{r}^{\prime}\right)+\left(\frac{f_{y_{r}^{\prime}}}{\eta}\left(x, y_{r},{ }_{c} D^{\beta} y_{r}, y_{r}^{\prime}\right)\right. \\
& \left.+f_{y_{r}^{\beta}}\left(x, y_{r},{ }_{c} D^{\beta} y_{r}, y_{r}^{\prime}\right) \frac{x^{1-\beta}}{\eta \Gamma(2-\beta)}+f_{y_{r}}\left(x, y_{r},{ }_{c} D^{\beta} y_{r}, y_{r}^{\prime}\right) \frac{x}{\eta}\right)\left(\gamma_{2}-\gamma_{1}\right) \\
& +f_{y_{r}}\left(x, y_{r},{ }_{c} D^{\beta} y_{r}, y_{r}^{\prime}\right) \gamma_{1}, \\
& g_{r}\left(x, y_{r},{ }_{c} D^{\beta} y_{r}, y_{r}^{\prime}\right) \\
= & \frac{f_{y_{r}^{\prime}}}{\eta}\left(x, y_{r}, D_{c} D^{\beta} y_{r}, y_{r}^{\prime}\right)+f_{y_{r}^{\beta}}\left(x, y_{r},{ }_{c} D^{\beta} y_{r}, y_{r}^{\prime}\right) \frac{x^{1-\beta}}{\eta \Gamma(2-\beta)} \\
& +f_{y_{r}}\left(x, y_{r},{ }_{c} D^{\beta} y_{r}, y_{r}^{\prime}\right) \frac{x}{\eta} .
\end{aligned}
$$

Equation (5.17) at the collocation points $x_{c}(j)=\eta \frac{2 j-1}{2^{k} M}, j=1,2, \ldots, \hat{m}$, and in vector notation, takes the following form by using equations (3.5), (3.9) 
and $(3.12)$

$$
\begin{gathered}
\mathbf{a}^{\lambda, r+1^{T}}\left(\Psi_{\hat{m} \times \hat{m}}^{\lambda}-\mathbf{F}_{\mathbf{r}}^{\prime} \mathbf{P}_{\hat{m} \times \hat{m}}^{\lambda, \alpha-1} \Psi_{\hat{m} \times \hat{m}}^{\lambda}-\mathbf{F}_{\mathbf{r}}^{\beta} \mathbf{P}_{\hat{m} \times \hat{m}}^{\lambda, \alpha-\beta} \Psi_{\hat{m} \times \hat{m}}^{\lambda}\right. \\
\left.-\mathbf{F}_{\mathbf{r}} \mathbf{P}_{\hat{m} \times \hat{m}}^{\lambda, \alpha} \Psi_{\hat{m} \times \hat{m}}^{\lambda}+\mathbf{Q}_{\hat{\mathbf{m}} \times \hat{\mathbf{m}}}^{\mathbf{g}_{\mathbf{m}}, \lambda, \alpha, \eta}\right)=\mathbf{D}^{r},
\end{gathered}
$$

where $\mathbf{a}^{\lambda, r+1}=\left[a_{1}^{\lambda, r+1}, a_{2}^{\lambda, r+1}, \ldots, a_{\hat{m}}^{\lambda, r+1}\right]$ is an unknown vector,

$$
\mathbf{D}^{r}=\left[\left.Q_{r}\right|_{x_{c}(1)},\left.\left.Q_{r}\right|_{x_{c}(2)} \cdots Q_{r}\right|_{x_{c}(\hat{m})}\right]
$$

is known vector. We get $\mathbf{a}^{\lambda, r+1}$, for each $r \geq 0$, from equation (5.18) and use it to obtain approximate values of $y_{r+1}(x), y_{r+1}^{\prime}(x),{ }_{c} D^{\beta} y_{r+1}(x)$ and ${ }_{c} D^{\alpha} y_{r+1}(x)$ at the collocation points.

Test problem: Consider the following $\alpha^{\text {th }}$ order fractional nonlinear Lane Emden boundary value problem

$(5.19){ }^{c} D^{\alpha} y(x)+\frac{2}{x} y^{\prime}(x)-6 y^{2}(x)=6+\frac{2}{x}-6\left(x^{2}+x\right)^{2}, 1<\alpha \leq 2,0 \leq x \leq 1$, subject to the boundary conditions

$$
y(0)=0, y(1)=2 .
$$

The exact solution, when $\alpha=2$, is given by $y(x)=x^{2}+x$.
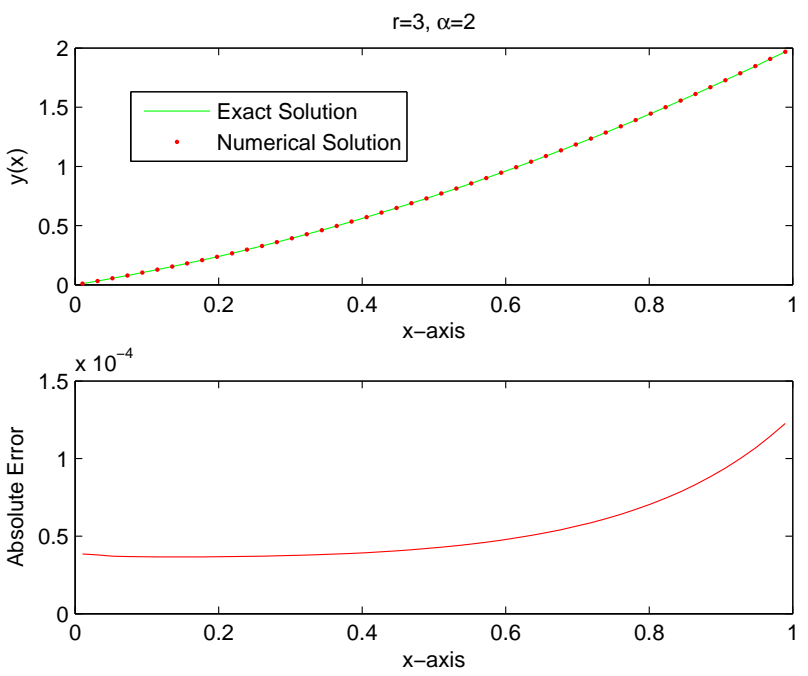

FIGURE 7. Solution by the Gegenbauer wavelet quasilinearization method by fixing $k=5, M=3, \lambda=9$, at $\alpha=2$ and $r=3$.

Implement the Gegenbauer wavelet quasilinearization technique to equation (5.19) to get Figures 7 and 8 . We fix the level of resolution, $k=5$, order of 


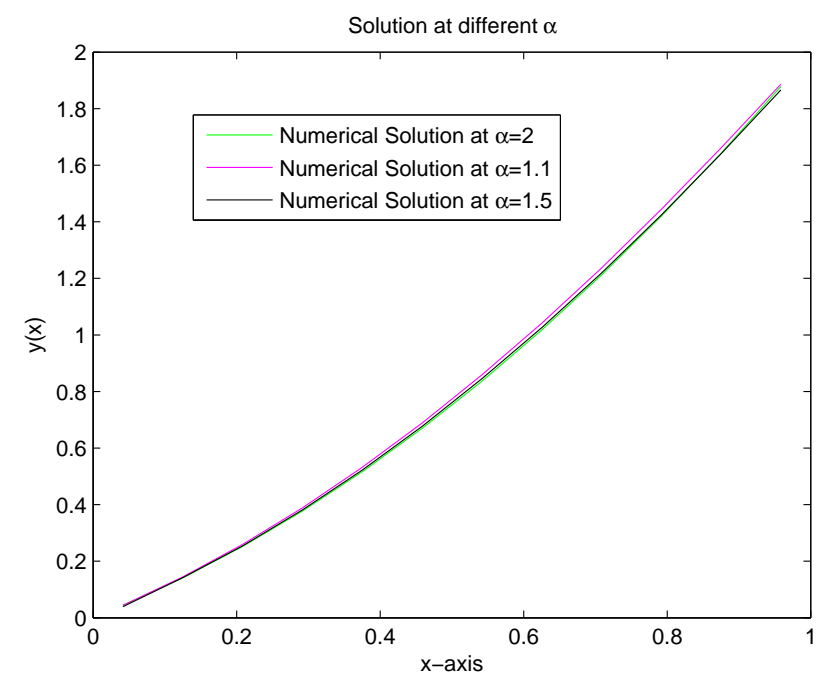

FiguRE 8 . Solution by the Gegenbauer wavelet quasilinearization method by using $k=3, M=3, \lambda=17.5$, at different values of $\alpha$ and $r=5$.

Gegenbauer polynomial, $M=3$, with $\lambda=9$ and order of differential equation (5.19), $\alpha=2$. The exact solution and solution by Gegenbauer wavelet quasilinearization technique at $r=3$, are shown in Figure 7 along with the absolute error.

Figure 8 is used to plot the solution by Gegenbauer wavelet quasilinearization technique at different values of $\alpha$ by fixing $\lambda=17.5, M=3, r=5$ and $k=3$.

\section{Conclusion}

We have derived the Gegenbauer wavelets matrix, $\Psi^{\lambda}{ }_{2^{k-1} M, 2^{k-1} M}$, the Gegenbauer wavelets operational matrix of fractional order integration, $\mathbf{P}_{\hat{m} \times \hat{m}}^{\lambda, \alpha}$, and another Gegenbauer wavelets operational matrix of fractional order integration, $\mathbf{Q}_{\hat{\mathbf{m}} \times \hat{\mathbf{m}}}^{\mathbf{g}, \lambda, \alpha, \mathbf{b}}$, which is used for solving boundary value problems. These matrices are successfully utilized to solve the fractional initial and boundary value problems with constant or variable coefficients.

According to the Table 1, we get more accurate results while increasing $k$ or $M$ or both, as in convergence analysis. The solution of the fractional order differential equation converge to the solution of integer order differential equation as in Figure 2. Gegenbauer wavelets method is also compared with the other numerical methods. Gegenbauer wavelets method is more accurate than the homotopy perturbation method and Haar wavelet method, as shown 
in Table 2. Gegenbauer wavelet method is highly competitive in comparison with the classical methods.

It is shown that Gegenbauer wavelet quasilinearization technique gives excellent results when applied to different fractional nonlinear initial boundary value problems. The results obtained from Gegenbauer wavelet quasilinearization technique are in good agreement with exact solutions, as shown in Figures 5 and 7 . The solution of the fractional nonlinear differential equation converge to the solution of the integer nonlinear differential equation as shown in Figures 6 and 8 . Different type of non-linearities can easily be handled by the Gegenbauer wavelet quasilinearization technique.

\section{References}

[1] A. Ali, M. A. Iqbal, and S. T. Mohyud-Din, Hermite wavelets method for boundary value problems, Int. J. Mod. Appl. Phys. 3 (2013), no. 1, 38-47.

[2] A. Arikoglu and I. Ozkol, Solution of fractional differential equations by using differential transform method, Chaos Solitons Fractals 34 (2007), no. 5, 1473-1481.

[3] E. Babolian and F. Fattahzdeh, Numerical solution of differential equations by using Chebyshev wavelet operational matrix of integration, Appl. Math. Comput. 188 (2007), no. 1, 417-426.

[4] R. E. Bellman, Functional equations in the theory of dynamic programming. II. Nonlinear differential equations, Proc. Natl. Acad. Sci. 41 (1955), 482-485.

[5] - Functional equations in the theory of dynamic programming. V. Positivity and quasilinearity, Proc. Natl. Acad. Sci. 41 (1955), 743-746.

[6] R. E. Bellman and R. E. Kalaba, Quasilinearization and nonlinear boundary-value problems, American Elsevier Publishing Company, 1965.

[7] C. Chen and C. Hsiao, Haar wavelet method for solving lumped and distributedparameter systems, IEE P.-Contr. Theor. Appl. 144 (1997), 87-94.

[8] I. Daubechies, Orthonormal bases of compactly supported wavelets, Comm. Pure Appl. Math. 41 (1988), no. 7, 909-996.

[9] M. Dehghan and M. Lakestani, Numerical solution of nonlinear system of second-order boundary value problems using cubic B-spline scaling functions, Int. J. Comput. Math. 85 (2008), no. 9, 1455-1461.

[10] J. V. Devi, F. A. McRae, and Z. Drici, Generalized quasilinearization for fractional differential equations, Comput. Math. Appl. 59 (2010), no. 3, 1057-1062.

[11] J. V. Devi and C. Suseela, Quasilinearization for fractional differential equations, Commun. Appl. Anal. 12 (2008), no. 4, 407-418.

[12] K. T. Elgindy and K. A. Smith-Miles, Solving boundary value problems, integral, and integro-differential equations using Gegenbauer integration matrices, J. Comput. Appl. Math. 237 (2013), no. 1, 307-325.

[13] A. E. M. El-Mesiry, A. M. A. El-Sayed, and H. A. A. El-Saka, Numerical methods for multi-term fractional (arbitrary) orders differential equations, Appl. Math. Comp. 160 (2005), no. 3, 683-699.

[14] S. A. El-Wakil, A. Elhanbaly, and M. A. Abdou, Adomian decomposition method for solving fractional nonlinear differential equations, Appl. Math. Comput. 182 (2006), no. 1, 313-324.

[15] N. Engheta, On fractional calculus and fractional multipoles in electromagnetism, IEEE T. Antenn. Propag. 44 (1996), no. 4, 554-566.

[16] I. Hashim, O. Abdulaziz, and S. Momani, Homotopy analysis method for fractional IVPs, Commun. Nonlinear Sci. Numer. Simul. 14 (2009), no. 3, 674-684. 
[17] E. Hesameddini, S. Shekarpaz, and H. Latifizadeh, The Chebyshev wavelet method for numerical solutions of a fractional oscillator, Int. J. Appl. Math. Research 1 (2012), no. 4, 493-509.

[18] A. Kilicman and Z. A. A. Al Zhour, Kronecker operational matrices for fractional calculus and some applications, Appl. Math. Comp. 187 (2007), no. 1, 250-265.

[19] V. V. Kulish and J. L. Lage, Application of fractional calculus to fluid mechanics, J. Fluids Eng. 124 (2002), 803-806.

[20] C. Lederman, J.-M. Roquejoffre, and N. Wolanski, Mathematical justification of a nonlinear integrodifferential equation for the propagation of spherical flames, Ann. Mat. Pura Appl. 183 (2004), no. 2, 173-239.

[21] U. Lepik, Solving PDEs with the aid of two-dimensional Haar wavelets, Comput. Math. Appl. 61 (2011), no. 7, 1873-1879.

[22] Y. Li, Solving a nonlinear fractional differential equation using Chebyshev wavelets, Commun. Nonlinear Sci. Numer. Simul. 15 (2010), no. 9, 2284-2292.

[23] R. L. Magin, Fractional calculus models of complex dynamics in biological tissues, Comput. Math. Appl. 59 (2010), no. 5, 1586-1593.

[24] F. Mainardi, Fractional calculus: some basic problems in continuum and statistical mechanics, in: A. Carpinteri, F. Mainardi (Eds.), Fractals and Fractional Calculus in Continuum Mechanics, pp. 291-348, Springer-Verlag, New York, 1997.

[25] M. Meerschaert and C. Tadjeran, Finite difference approximations for two-sided spacefractional partial differential equations, Appl. Numer. Math. 56 (2006), no. 1, 80-90.

[26] R. N. Mohapatra, K. Vajravelu, and Y. Yin, An improved quasilinearization method for second order nonlinear boundary value problems, J. Math. Anal. Appl. 214 (1997), no. $1,55-62$.

[27] C. A. Monje, Y. Q. Chen, B. M. Vinagre, D. Xue, and V. Feliu, Fractional-order systems and controls, Advances in Industrial Control, Springer, 2010.

[28] M. Razzaghi and S. Yousefi, The Legendre wavelets operational matrix of integration, Internat. J. Systems Sci. 32 (2001), no. 4, 495-502.

[29] _ Legendre wavelets method for constrained optimal control problems, Math. Methods Appl. Sci. 25 (2002), no. 7, 529-539.

[30] M. Rehman and R. A. Khan, A numerical method for solving boundary value problems for fractional differential equations, Appl. Math. Model. 36 (2012), no. 3, 894-907.

[31] U. Saeed and M. Rehman, Haar wavelet-quasilinearization technique for fractional nonlinear differential equations, Appl. Math. Comput. 220 (2013), 630-648.

[32] W Wavelet-Galerkin quasilinearization method for nonlinear boundary value problems, Abstr. Appl. Anal. 2014 (2014), Article ID 868934, 10 pages.

[33] _ Hermite wavelet method for fractional delay differential equations, J. Difference Equations 2014 (2014), Article ID 359093, 8 pages.

[34] L. R. Soares, H. M. de Oliveira, and R. J. D. Sobral Cintra, New compactly supported scaling and wavelet functions derived from Gegenbauer polynomials, Electrical and Computer Engineering, Canadian Conference on 2-5 May 2004 (vol.4) (2004), 2347-2350; DOI: 10.1109/CCECE.2004.1347717.

[35] S. G. Venkatesh, S. K. Ayyaswamy, and S. R. Balachandar, The Legendre wavelet method for solving initial value problems of Bratu-type, Comput. Math. Appl. 63 (2012), no. 8, $1287-1295$.

[36] Y. Wang and Q. Fan, The second kind Chebyshev wavelet method for solving fractional differential equations, Appl. Math. Comp. 218 (2012), no. 17, 8592-8601.

[37] Y. Wang, H. Song, and D. Li, Solving two-point boundary value problems using combined homotopy perturbation method and Greens function method, Appl. Math. Comput. 212 (2009), no. 2, 366-376 
Mujeeb ur ReHman

School of Natural Sciences

National University of Sciences and Technology

Sector H-12 Islamabad Pakistan

E-mail address: mujeeburrehman345@yahoo.com

UMER SAEED

School of Natural Sciences

National University of Sciences and Technology

SeCtor H-12 Islamabad PAKISTAN

E-mail address: umer.math@gmail.com 